MATHEMATICS OF COMPUTATION

Volume 72, Number 242, Pages 619-656

S 0025-5718(02)01462-X

Article electronically published on November 4, 2002

\title{
MAXIMUM NORM STABILITY OF DIFFERENCE SCHEMES FOR PARABOLIC EQUATIONS ON OVERSET NONMATCHING SPACE-TIME GRIDS
}

\author{
T. P. MATHEW AND G. RUSSO
}

\begin{abstract}
In this paper, theoretical results are described on the maximum norm stability and accuracy of finite difference discretizations of parabolic equations on overset nonmatching space-time grids. We consider parabolic equations containing a linear reaction term on a space-time domain $\Omega \times[0, T]$ which is decomposed into an overlapping collection of cylindrical subregions of the form $\Omega_{l}^{*} \times[0, T]$, for $l=1, \ldots, p$. Each of the space-time domains $\Omega_{l}^{*} \times[0, T]$ are assumed to be independently grided (in parallel) according to the local geometry and space-time regularity of the solution, yielding space-time grids with mesh parameters $h_{l}$ and $\tau_{l}$. In particular, the different space-time grids need not match on the regions of overlap, and the time steps $\tau_{l}$ can differ from one grid to the next. We discretize the parabolic equation on each local grid by employing an explicit or implicit $\theta$-scheme in time and a finite difference scheme in space satisfying a discrete maximum principle. The local discretizations are coupled together, without the use of Lagrange multipliers, by requiring the boundary values on each space-time grid to match a suitable interpolation of the solution on adjacent grids. The resulting global discretization yields a large system of coupled equations which can be solved by a parallel Schwarz iterative procedure requiring some communication between adjacent subregions. Our analysis employs a contraction mapping argument.

Applications of the results are briefly indicated for reaction-diffusion equations with contractive terms and heterogeneous hyperbolic-parabolic approximations of parabolic equations.
\end{abstract}

\section{INTRODUCTION}

In this paper, theoretical bounds are described (extending results in [32, 10]) for the maximum norm stability and convergence of discretizations of parabolic equations on nonmatching, overset space-time grids. Nonmatching overset spatial grids are popular in several fluid dynamics computations involving complex geometries [33, 13]. They permit independent (parallel) generation of local grids adapted to the local geometry (without the restriction of matching the grids on the regions of overlap) at the cost of increased computations in coupling the various local discretizations. For evolution problems, additional flexibility can be obtained

Received by the editor July 25, 2000.

2000 Mathematics Subject Classification. Primary 65N20, 65F10.

Key words and phrases. Nonmatching overset space-time grids, maximum norm stability, composite grids, parallel Schwarz alternating method, parabolic equations, discrete maximum principle, discrete barrier functions.

(C)2002 American Mathematical Society 
by permitting different time steps and choice of explicit or implicit schemes on each of the different space-time subregions [33, 13, 17, 18, 7, 23, 5, 7, 20, 22.

In the computational literature, several approaches have been proposed for coupling discretizations on nonmatching grids. These include Lagrange multipliers (including mortar methods) and least squares based techniques (see [33, 13, 32, 5 4, 9, 23, 20, 1, 2, 10, 19). The method considered in this paper does not use either Lagrange multipliers or least squares to couple the various local problems. It is simpler to implement (see [32, 10]); however, it applies only to a certain class of parabolic equations exhibiting a contraction property, and it requires overlap amongst adjacent grids.

Our study will be restricted to a small class of parabolic equations of the form

$$
\left\{\begin{aligned}
u_{t}-a \Delta u+\vec{b}(x) \cdot \nabla u+c(x) u & =f(x, t), & & \text { in } \Omega \times[0, T] \\
u(x, t) & =0, & & \text { on } \partial \Omega \times[0, T], \\
u(x, 0) & =u_{0}(x), & & \text { on } \Omega,
\end{aligned}\right.
$$

where $f(x, t), \vec{b}(x), c(x)$ and $u_{0}(x)$ are sufficiently smooth functions and $a>0$. Here $\Omega \subset R^{d}$ for $d=1,2, \ldots$ In order to have a contraction property for homogeneous solutions, we will require that

$$
c(x) \geq c_{0}>0,
$$

for some positive constant $c_{0}$.

Given the cylindrical space-time domain $\Omega \times[0, T]$, we decompose it into an overlapping collection of cylinders of the form $\left\{\Omega_{l}^{*} \times[0, T]\right\}_{l=1}^{p}$ that form a covering of $\Omega \times[0, T]$. Each cylinder $\Omega_{l}^{*} \times[0, T]$ will be assumed to be triangulated by a space-time grid with mesh and time parameters $h_{l}$ and $\tau_{l}$ (see Figure 1.1). We employ finite difference methods in space and implicit or explicit $\theta$-schemes in time, independently on each space-time grid. On each subdomain boundary $\partial \Omega_{l}^{*} \times[0, T]$, we require the local solution to match some suitably chosen interpolant of the solution from adjacent grids (see [33, 32, 13, 10]).

Our main result in the paper, stated in Theorem 4.4, concerns the accuracy of the global discretization. Let $u_{h, \tau}$ denote the restriction of the exact solution $u$ of the parabolic equation to all the space-time gridpoints, and let $U_{h, \tau}$ denote the computed solution of the global discretization. Suppose the truncation and intergrid interpolation errors for the discretization and boundary conditions, respectively, on the $l$ th space-time grid $\Omega_{l}^{*} \times[0, T]$ satisfy

Local truncation error on $\Omega_{l}^{*} \times[0, T]=\|u\|_{q_{l ; 1}+2, q_{l ; 2}+2, \infty, \Omega_{l}^{*} \times[0, T]}\left(h_{l}^{q_{l ; 1}}+\tau_{l}^{q_{l ; 2}}\right)$, Local interpolation error on $\partial \Omega_{l}^{*} \times[0, T]=\|u\|_{r_{l ; 1}, r_{l ; 2}, \infty, B_{2}^{l, *} \times[0, T]}\left(h_{l}^{r_{l ; 1}}+\tau_{l}^{r_{l ; 2}}\right)$, where $\|\cdot\|_{q-l ; 1, q-l ; 2, \infty, \Omega_{l}^{*} \times[0, T]}$ and $\|\cdot\|_{r-l ; 1, r-l ; 2, \infty, B_{2}^{l, *} \times[0, T]}$ denote Sobolev norms. Here $B_{2}^{l, *}$ is a small spatial region covering the the boundary segment $B_{2}^{l}=$ $\left(\partial \Omega_{l}^{*} \cap \Omega\right)$ of the $l$ th spatial subdomain. Theorem 4.4 states that, under suitable assumptions, the maximum norm of the global error $u_{h, \tau}-U_{h, \tau}$ satisfies the bound

$$
\begin{aligned}
\left\|\left|u_{h, \tau}-U_{h, \tau}\right|\right\| \leq C \max _{l}\left\{\|u\|_{q-l ; 1+2, q-l ; 2+2, \infty, \Omega_{l}^{*} \times[0, T]}\left(h_{l}^{q_{l ; 1}}+\tau_{l}^{q_{l ; 2}}\right)\right. \\
\left.+\|u\|_{r_{l ; 1}, r_{l ; 2}, \infty, B_{2}^{l, *} \times[0, T]}\left(h_{l}^{r_{l ; 1}}+\tau_{l}^{r_{l ; 2}}\right)\right\},
\end{aligned}
$$

where $C>0$ is independent of the mesh sizes. From this we deduce that, ideally, the local grid sizes $h_{l}$ and $\tau_{l}$ should be chosen so that all the local error terms are "balanced". This would mean smaller $h_{l}$ and $\tau_{l}$ on regions where the solution is 
Overlapping space-time subregions. Nonmatching overset grids.
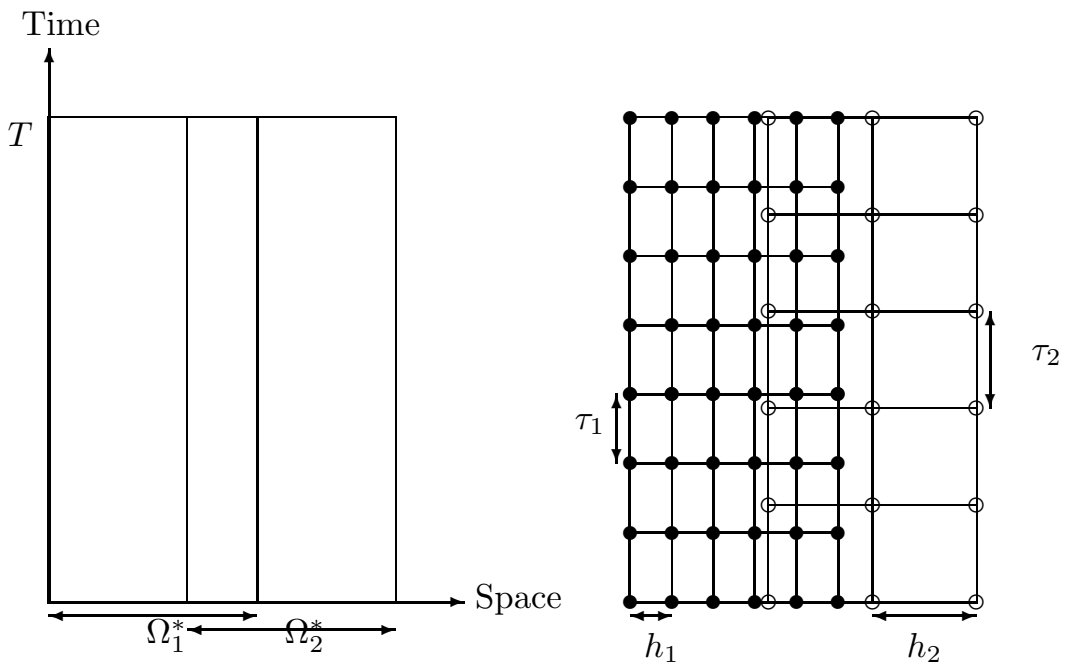

Figure 1.1. Sample nonmatching overset space-time grid.

less regular. Additionally, the intergrid interpolation maps for determining local boundary data should be chosen so that the interpolation errors are balanced with the local truncation errors. Then, the resulting accuracy of the global discretization will be of optimal order.

The rest of the paper is outlined as follows. In Section 2, we introduce notation for the overlapping space-time subregions, discuss explicit and implicit local discretizations on each space-time grid, intergrid interpolation maps, the global discretization, and a parallel Schwarz iterative procedure for solving the resulting system of equations. In Section 3, we discuss theoretical properties of the local schemes, such as a priori estimates, maximum principles, comparison theorems, barrier functions, and contraction properties of homogeneous solutions. In Section 4, we analyze the stability and accuracy of our global space-time discretization by employing Picard's contraction mapping theorem.

\section{Global Discretization}

\section{ON NONMATCHING OVERLAPPING SPACE-TIME GRIDS}

In this section, we describe the construction of a global discretization of (1.1) and a parallel Schwarz iterative method for solving the resulting large system of equations.

2.1. Space-time subdomains. Let $\Omega \times[0, T]$ denote the space-time region on which the parabolic equation (1.1) is posed. We will describe here the construction of an overlapping collection of space-time subregions that covers the above region.

Let $\left\{\Omega_{l}\right\}_{l=1}^{p}$ denote a partition or a covering of the spatial domain $\Omega$ :

$$
\bar{\Omega} \subset \bigcup_{l=1}^{p} \bar{\Omega}_{l} .
$$


In practice, the subregions $\Omega_{l}$ may be chosen according to the geometry of $\Omega$ or the regularity of the solution (if known, or else by estimating the regularity from prior numerical approximations). For each subregion $\Omega_{l}$, choose a parameter $\beta_{l}>0$ and enlarge $\Omega_{l}$ to $\Omega_{l}^{*}$ as

$$
\Omega_{l}^{*} \equiv\left\{x \in \Omega: \operatorname{dist}\left(x, \Omega_{l}\right)<\beta_{l}\right\} .
$$

The collection of subregions $\left\{\Omega_{l}^{*}\right\}_{l=1}^{p}$ will form an overlapping covering of $\Omega$ (with overlap parameters $\beta_{l}$ ). An overlapping covering of the space-time region $\Omega \times[0, T]$ can be immediately constructed:

$$
\Omega \times[0, T]=\bigcup_{l=1}^{p}\left(\Omega_{l}^{*} \times[0, T]\right),
$$

where each space-time subregion $\Omega_{l}^{*} \times[0, T]$ is cylindrical.

We will denote the boundary of each spatial subregion $\Omega_{l}^{*}$ by $B^{l} \equiv \partial \Omega_{l}^{*}$. It will be convenient to further partition each boundary $B^{l}=\partial \Omega_{l}^{*}$ into two segments $B_{1}^{l}$ and $B_{2}^{l}$ (we will omit the superscript $l$ when the subregion is clear from the context):

$$
B_{1}^{l} \equiv \partial \Omega_{l}^{*} \cap \partial \Omega \quad \text { and } \quad B_{2}^{l} \equiv \partial \Omega_{l}^{*} \cap \Omega .
$$

Corresponding to this, the space-time boundary $B^{l} \times[0, T]$ of each local subregion can be decomposed into $B_{1}^{l} \times[0, T]$ and $B_{2}^{l} \times[0, T]$.

2.2. Local space-time grids. On each of the local space-time cylinders $\Omega_{l}^{*} \times[0, T]$, we assume that a space-time grid $\Omega_{h_{l}}^{*} \times\left\{0, \tau_{l}, 2 \tau_{l}, \ldots, T-\tau_{l}, T\right\}$ is constructed, taking into account the geometry of $\Omega_{l}^{*}$ and the regularity of the solution on this space-time region (see Figure [1.1). Here $h_{l}$ denotes the mesh size on $\Omega_{l}^{*}$ and $\tau_{l}$ denotes the time step with

$$
\tau_{l}=\frac{T}{N_{l}}
$$

for some integer $N_{l} \geq 1$. Throughout the paper, $x_{i}^{h_{l}}$ will denote the $i$ th gridpoint in $\bar{\Omega}_{h_{l}}^{*}$.

We will use $I^{h_{l}}$ to denote the interior nodes in the grid $\Omega_{h_{l}}^{*}$ and $B^{h_{l}}$ to denote its boundary nodes. Since $B^{l}$ is decomposed into $B_{1}^{l}$ and $B_{2}^{l}$, we denote by $B_{1}^{h_{l}}$ and $B_{2}^{h_{l}}$, the gridpoints on $B_{1}^{l}$ and $B_{2}^{l}$, respectively. We will denote a grid function on $\bar{\Omega}_{h_{l}}^{*}$ by $w_{h_{l}}$. Corresponding to the partition of gridpoints in $\bar{\Omega}_{h_{l}}^{*}$ into $I^{h_{l}}$ and $B^{h_{l}}$, we obtain the block vector

$$
w_{h_{l}}=\left(w_{h_{l}, I}, w_{h_{l}, B}\right)^{T} .
$$

When it is necessary to distinguish the block components of $w_{h_{l}}$ corresponding to the boundary subgrids $B_{1}^{h_{l}}$ and $B_{2}^{h_{l}}$, we will use the notation $w_{h_{l}, B_{1}}$ and $w_{h_{l}, B_{2}}$, respectively:

$$
w_{h_{l}, B}=\left(w_{h_{l}, B_{1}}, w_{h_{l}, B_{2}}\right)^{T} .
$$

We will denote a grid function on $\bar{\Omega}_{h_{l}}^{*}$ at time $k \tau_{l}$ by $w_{h_{l}}^{k}$. A space-time grid function on $\bar{\Omega}_{h_{l}}^{*} \times\left\{0, \tau_{l}, \ldots, T\right\}$ will be denoted by $w_{h_{l}, \tau_{l}}$ with

$$
w_{h_{l}, \tau_{l}}=\left\{w_{h_{l}}^{k}\right\}_{k=0}^{N_{l}} .
$$


A grid function on the entire family of space-time grids will be denoted by $w_{h, \tau}$, where

$$
\begin{aligned}
w_{h, \tau} & \equiv\left(w_{h_{l}, \tau_{l}}\right)_{l=1}^{p} \\
& =\left(\left\{w_{h_{l}}^{k}\right\}_{k=0}^{N_{l}}\right)_{l=1}^{p} .
\end{aligned}
$$

Given a continuous spatial function $w(x)$, we will use $\pi_{h_{l}} w$ to denote its spatial interpolation onto the gridpoints in $\bar{\Omega}_{h_{l}}^{*}$

$$
\left(\pi_{h_{l}} w\right)_{i} \equiv w\left(x_{i}^{h_{l}}\right), \quad x_{i}^{h_{l}} \in \bar{\Omega}_{h_{l}}^{*} .
$$

Similarly, $\pi_{h_{l}, I} w$ will denote interpolation of $w(x)$ onto the interior gridpoints in $\Omega_{h_{l}}^{*}$. If $w(x, t)$ is a continuous space-time function, we will use $\pi_{h_{l}, \tau_{l}}$ to denote the interpolation of $w(.,$.$) onto the l$ th space-time grid

$$
\pi_{h_{l}, \tau_{l}} w \equiv\left\{\pi_{h_{l}} w\left(., k \tau_{l}\right)\right\}_{k=0}^{N_{l}} .
$$

If $w(x, t)$ is a continuous space-time function, we will use $\pi_{h, \tau}$ to denote the interpolation of $w(.,$.$) onto all the space-time grids$

$$
\pi_{h, \tau} w \equiv\left(\pi_{h_{l}, \tau_{l}} w\right)_{l=1}^{p} .
$$

2.3. Local subproblems and local discretizations. On each of the space-time subdomains $\Omega_{l}^{*} \times[0, T]$, the original parabolic equation (1.1) will be replaced by the following local parabolic initial boundary value problem with suitably chosen boundary conditions $g_{l}(x, t)$ that couple the adjacent problems:

$$
\begin{aligned}
u_{t}+L u & =f(x, t), & & (x, t) \in \Omega_{l}^{*} \times[0, T], \\
u & =0, & & (x, t) \in B_{1}^{l} \times[0, T], \\
u & =g_{l}(x, t), & & (x, t) \in B_{2}^{l} \times[0, T], \\
u(x, 0) & =u_{0}(x), & & t=0,
\end{aligned}
$$

where $L$ denotes the elliptic operator

$$
L u \equiv-a \Delta u+\vec{b} \cdot \nabla u+c(x) u .
$$

Here, the choice of local initial data is $u_{0}(x)$ restricted to $\Omega_{l}^{*}$ since the exact solution restricted to $\Omega_{l}^{*} \times[0, T]$ would satisfy this initial condition and since $u_{0}(x)$ is assumed to be known. The boundary data on $B_{1}^{l} \times[0, T]$ is zero, since the exact solution satisfies this boundary condition. The boundary data $g_{l}(x, t)$ will play a crucial role, as it is not known. We will require (see subsection 2.5) that $g_{l}(x, t)$ equals a suitable interpolation of the solutions from adjacent regions. This will couple the various local problems and require an iterative process to compute $g_{l}(x, t)$.

Each local parabolic equation will be discretized on the space-time grid $\Omega_{h_{l}}^{*} \times$ $\left\{0, \tau_{l}, \ldots, T\right\}$ by a finite difference scheme in space and an implicit or explicit $\theta$ scheme in time. The elliptic operator $L$ will be discretized on each spatial grid $\Omega_{h_{l}}^{*}$ by a finite difference scheme with coefficient matrix $A^{h_{l}}$. If $x_{i}^{h_{l}}$ is the $i$ th interior gridpoint in $\Omega_{h_{l}}^{*}$, then the discretization of $L$ at this gridpoint will be denoted by

$$
L w\left(x_{i}^{h_{l}}\right)=\sum_{j} A_{i j}^{h_{l}} w\left(x_{j}^{h_{l}}\right)+\mathcal{C}_{h_{l}}\left(w, x_{i}^{h_{l}}\right),
$$

where $\mathcal{C}\left(w, x_{i}^{h_{l}}\right)$ is the local truncation error at $x_{i}^{h_{l}}$ for an arbitrary smooth function $w(x)$. The matrix $A^{h_{l}}$ will be rectangular; the first index ( $i$ in the preceding 
equation) corresponds to interior gridpoints, while the second index ( $j$ above) corresponds to interior and/or boundary gridpoints. Corresponding to the partition $w_{h_{l}}=\left(w_{h_{l}, I}, w_{h_{l}, B}\right)^{T}$, the rectangular matrix $A^{h_{l}}$ can be block partitioned

$$
A^{h_{l}}=\left[\begin{array}{ll}
A_{I I}^{h_{l}} & A_{I B}^{h_{l}}
\end{array}\right], \quad \text { with } \quad A^{h_{l}} w_{h_{l}}=A_{I I}^{h_{l}} w_{h_{l}, I}+A_{I B}^{h_{l}} w_{h_{l}, B} .
$$

Assumption A1. We will assume the following about the entries of $A^{h_{l}}$.

1. $A_{i i}^{h_{l}}>0$ for all $i \in I^{h_{l}}$.

2. $A_{i j}^{h_{l}} \leq 0$ when $i \neq j$ with $i \in I^{h_{l}}$ and $j \in I^{h_{l}} \cup B^{h_{l}}$.

3. $\sum_{j} A_{i j}^{h_{l}}=c_{i}^{h_{l}} \geq c_{0}>0$.

Remark 1. Finite difference discretizations satisfying Assumption $A 1$ can be constructed in many ways. If the grid $\Omega_{h_{l}}^{*}$ is uniform, then the standard second-order five-point stencil may be applied to approximate $\sum_{i} \frac{\partial}{\partial x_{i}}\left(a \frac{\partial w}{\partial x_{i}}\right)$. If $a$ is not small in relation to $\|\vec{b}\|_{\infty, \Omega_{l}^{*}}$, then centered finite differences may be applied to obtain a second order approximation to $\vec{b}(x) \cdot \nabla w$, provided a local cell Peclet restriction (of the form $\|\vec{b}\|_{\infty, \Omega_{l}^{*}} h<2 a$ ) is satisfied. If $a$ is small in relation to $\|\vec{b}\|_{\infty, \Omega_{l}^{*}}$ (or zero), then a first order upwind discretization can be applied to approximate $\vec{b}(x) \cdot \nabla w$. The term $c(x) w(x)$ can be approximated by a one-point stencil at each gridpoint. If the grid is nonuniform, finite volume based finite differences may be applied to construct the desired approximations. For instance, if $\Omega \subset R^{2}$, then a Delaunay triangulation need first to be constructed for the grid and finite volume based finite differences can be applied (see [6] ).

Remark 2. If matrix $A^{h_{l}}$ satisfies Assumption $A 1$, then $A^{h_{l}}$ will be strictly diagonally dominant and $\left(\left(A_{I I}^{h_{l}}\right)^{-1}\right)_{i j} \geq 0$ for all $i, j$. In particular, $A_{I I}^{h_{l}}$ will be an $M$-matrix (see, for instance [31]).

Semi-discretization of the local parabolic initial boundary value problem on the spatial grid $\Omega_{h_{l}}^{*}$ yields

$$
\begin{aligned}
\frac{d U_{h_{l}, I}}{d t}+A_{I I}^{h_{l}} U_{h_{l}, I}+A_{I B}^{h_{l}} U_{h_{l}, B} & =f_{h_{l}, I}(t), \\
U_{h_{l}, B_{1}}(t) & =0, \\
U_{h_{l}, B_{2}}(t) & =g_{h_{l}, B_{2}}(t), \\
U_{h_{l}}(0) & =\pi_{h_{l}} u_{0}(x),
\end{aligned}
$$

where $f_{h_{l}, I}(t) \equiv \pi_{h_{l}, I} f(., t)$. The boundary conditions $g_{h_{l}, B_{2}}(t)$ will be specified in subsections 2.4 and 2.5 . 
To discretize (2.1) in time, we choose $0 \leq \theta_{l} \leq 1$, for $l=1, \ldots, p$ independently, and apply a $\theta$-scheme to obtain

$$
\begin{aligned}
\frac{U_{h_{l}, I}^{k+1}-U_{h_{l}, I}^{k}+\theta_{l} A_{I I}^{h_{l}} U_{h_{l}, I}^{k+1}+\theta_{l} A_{I B}^{h_{l}} U_{h_{l}, B}^{k+1}}{\tau_{l}} & \\
+\tilde{\theta}_{l} A_{I I}^{h_{l}} U_{h_{l}, I}^{k}+\tilde{\theta}_{l} A_{I B}^{h_{l}} U_{h_{l}, B}^{k} & =\theta_{l} f_{h_{l}, I}^{k+1}+\tilde{\theta}_{l} f_{h_{l}, I}^{k}, \\
U_{h_{l}, B_{1}}^{k+1} & =0, \\
U_{h_{l}, B_{2}}^{k+1} & =g_{h_{l}, B_{2}}^{k+1}, \\
U_{h_{l}, 0}^{0} & =\pi_{h_{l}, I} u_{0},
\end{aligned}
$$

for $k=0, \ldots, N_{l}-1$, where $\tilde{\theta}_{l} \equiv 1-\theta_{l}$. The discrete boundary conditions $\left\{g_{h_{l}, B_{2}}^{k}\right\}$ for $k=1, \ldots, N_{l}$ and $l=1, \ldots, p$ are crucial for coupling the various local parabolic discretizations and will be described in the next section.

2.4. Intergrid interpolation. The local space-time discretizations (2.2) will be coupled together by requiring that the local boundary data $g_{h_{l}, B_{2}}^{k}$ match a suitable interpolation $\mathcal{I}_{h_{l}}^{k} U_{h, \tau}$ of the discrete solution $U_{h, \tau}$ from adjacent grids. The linear map $\mathcal{I}_{h_{l}}^{k}$ is described below.

Let $x_{i}^{h_{l}}$ (from $\bar{\Omega}_{h_{l}}^{*}$ ) be a gridpoint on $B_{2}^{l}$. At time $k \tau_{l}$ the boundary data $\left(g_{h_{l}, B_{2}}^{k}\right)_{i}=\left(U_{h_{l}}^{k}\right)_{i} \approx u\left(x_{i}^{h_{l}}, k \tau_{l}\right)$ will be approximated by linear combinations of nodal values of $\left(U_{h_{\tilde{l}}}^{\tilde{k}}\right)_{j} \approx u\left(x_{j}^{h_{\tilde{\tau}}}, \tilde{k} \tau_{\tilde{l}}\right)$ from adjacent space-time grids $\bigcup_{\tilde{l} \neq l} \bar{\Omega}_{\tilde{l}} \times[0, T]$ with

$$
\left(U_{h_{l}}^{k}\right)_{i}=\left(\mathcal{I}_{h_{l}}^{k} U_{h, \tau}\right)_{i},
$$

where the interpolation map $\mathcal{I}_{h_{l}}^{k}$ is defined in terms of a tensor $\alpha_{\tilde{l}, \tilde{k}, \tilde{i}}^{l, k, i}$ :

$$
\left(\mathcal{I}_{h_{l}}^{k} U_{h, \tau}\right)_{i} \equiv \sum_{\tilde{l}=1}^{p} \sum_{\tilde{k}=0}^{N_{\tilde{l}}} \sum_{\tilde{i}} \alpha_{\tilde{l}, \tilde{k}, \tilde{i}}^{l, k, i}\left(U_{h_{\tilde{l}}}^{\tilde{k}}\right)_{\tilde{i}} .
$$

Below we list assumptions about the weights $\alpha_{\tilde{l}, \tilde{k}, \tilde{i}}^{l, k, i}$ used to define $\left(\mathcal{I}_{h_{l}}^{k} U_{h, \tau}\right)_{i}$.

1. Assumption A2. The intergrid interpolation map $\mathcal{I}_{h_{l}}^{k}$ must use only values from adjacent grids $\tilde{l} \neq l$. In terms of the coefficients, this places the following requirement on the weights $\alpha_{\tilde{l}, \tilde{k}, \tilde{i}}^{l, k, i}$ :

$$
\alpha_{\tilde{l}, \tilde{k}, \tilde{i}}^{l, k, i}=0, \quad \text { when } l=\tilde{l} .
$$

2. Assumption A3. Given $l$, the intergrid interpolation map $\mathcal{I}_{h_{l}}^{k}$ should involve only nodal values from gridpoints in the unextended subregions $\bar{\Omega}_{\tilde{l}} \times[0, T]$ for $\tilde{l} \neq l$, i.e., it should not involve nodal values from gridpoints in the extended regions $\bigcup_{\tilde{l} \neq l}\left(\Omega_{\tilde{l}}^{*} \backslash \bar{\Omega}_{\tilde{l}}\right) \times[0, T]$. In terms of the coefficients $\alpha_{\tilde{l}, \tilde{k}, \tilde{i}}^{l, k, i}$ this requirement is

$$
\alpha_{\tilde{l}, \tilde{k}, \tilde{i}}^{l, k, i}=0, \quad \text { when } x_{j}^{h_{\tilde{l}}} \in \Omega_{\tilde{l}}^{*} \backslash \bar{\Omega}_{\tilde{l}} \text { or } \tilde{l}=l .
$$


Given $B_{2}^{l}$, let $B_{2}^{l, *} \subset \Omega$ denote the smallest region such that $B_{2}^{l, *} \times[0, T]$ contains all the cells and gridpoints from adjacent subregions used in defining the interpolation map $\mathcal{I}_{h_{l}}^{k}$ for $k=0, \ldots, N_{l}$.

3. Assumption A4. Let $w(x, t)$ be a smooth space-time function which is zero on $\partial \Omega \times[0, T]$. For each space-time gridpoint $\left(x_{j}^{h_{l}}, k \tau_{l}\right)$ on the boundary segment $B_{2}^{l} \times[0, T]$ we assume that the interpolation is chosen so that the error satisfies

$$
w\left(x_{j}^{h_{l}}, k \tau_{l}\right)-\left(\mathcal{I}_{h_{l}}^{k} w_{h, \tau}\right)_{j}=\mathcal{D}_{h_{l}}^{k}\left(w, x_{j}^{h_{l}}\right),
$$

where the local interpolation error can be estimated by Taylor series expansion

$$
\left|\mathcal{D}_{h_{l}}^{k}\left(w, x_{j}^{h_{l}}\right)\right| \leq C\|w\|_{r_{l, 1}, r_{l, 2}, \infty, B_{2}^{l, *} \times[0, T]}\left(h_{l}^{r_{l, 1}}+\tau_{l}^{r_{l, 2}}\right),
$$

for some integers $r_{l, 1} \geq 1$ and $r_{l, 2} \geq 1$, where $C$ is independent of $h_{l}, \tau_{l}$ and $\|w\|_{r_{l, 1}, r_{l, 2}, \infty, B_{2}^{l, *} \times[0, T]}$ denotes a Sobolev norm of the space-time function $w(.,$.$) on the region B_{2}^{l, *} \times[0, T]$.

Definition. Throughout the paper, we will use $\sigma_{h, \tau}$ to denote the maximum norm of the intergrid interpolation map

$$
\sigma_{h, \tau} \equiv \max _{l, k, i} \sum_{\tilde{l}=1}^{p} \sum_{\tilde{k}=0}^{N_{\tilde{l}}} \sum_{\tilde{i}}\left|\alpha_{\tilde{l}, \tilde{k}, \tilde{i}}^{l, k, i}\right| .
$$

Example. We include a simple example to illustrate the intergrid interpolation map for a one-dimensional region $\Omega=(0,4)$ with $T=1$ and a two-subdomain decomposition with $\Omega_{1}=(0,2)$ and $\Omega_{2}=(2,4)$. Let the overlap parameters be $\beta_{l}=1$ for $l=1,2$. Then $\Omega_{1}^{*}=(0,3)$ and $\Omega_{2}^{*}=(1,4)$. Let the space-time grids be chosen with $h_{1}=3 / 10, \tau_{1}=1 / 10, h_{2}=3 / 4$ and $\tau_{2}=1 / 3$. Let the gridpoints be $x_{i}^{h_{1}}=i h_{1}$ for $i=0, \ldots, 10$ in $\Omega_{h_{1}}^{*}$ and $x_{i}^{h_{2}}=1+i h_{2}$ for $i=0, \ldots, 4$. We will consider a second-order accurate interpolation scheme. In this example, $B_{1}^{h_{1}}=0$, $B_{2}^{h_{1}}=3, B_{1}^{h_{2}}=4$ and $B_{2}^{h_{2}}=1$. The space-time gridpoints on $B_{2}^{1} \times[0,1]$ are $\{(3, k / 10): k=0, \ldots, 10\}$. We will describe how the entries of the map $\mathcal{I}_{h_{l}}^{k}$ can be constructed for defining the interpolated values at $\left(x_{i}^{h_{l}}, k \tau_{l}\right)$ for $k=1, l=1$ and $i=10$. The other grid values can be constructed similarly. The boundary gridpoint $(3,1 / 10)$ on boundary $B_{2}^{1} \times[0,1]$ is enclosed in the cell with vertices $(2.5,0),(3.25,0)(2.5,1 / 3)$ and $(3.25,1 / 3)$ (whose vertices are all gridpoints in the space-time subdomain $\left.\Omega_{2}^{*} \times[0,1]\right)$. Note that these four nodes are contained in $\bar{\Omega}_{2} \times[0,1]$. We will define our approximation to $\left(U_{h_{1}}^{1}\right)_{10}$ by using (second-order) bilinear interpolation:

$$
\left(U_{h_{1}}^{1}\right)_{10}=\frac{23}{90} U_{h_{2}}(2.5,0)+\frac{46}{90} U_{h_{2}}(3.25,0)+\frac{7}{90} U_{h_{2}}(2.5,1 / 3)+\frac{14}{90} U_{h_{2}}(3.25,1 / 3) .
$$

The interpolation map can be defined similarly for the other gridpoints on $B_{2}^{h_{1}} \times$ $[0,1]$ so that Assumptions $A 2$ and $A 3$ are satisfied. If all the stencils involve convex combinations (as in the above stencil), then the interpolation map will have maximum norm $\sigma_{h, \tau}=1$. In this example, the interpolation error is second order in the mesh parameters $h_{2}$ and $\tau_{2}$ of the space-time grid on $\Omega_{2}^{*} \times[0, T]$ with coefficients 
that depend on second derivatives of the function being interpolated (in the convex hull of the four nodes involved in the stencil):

$$
\begin{gathered}
\mid w\left(x_{10}^{h_{1}}, \tau_{1}\right)-\left(\frac{23}{90} W_{h_{2}}(2.5,0)+\frac{46}{90} W_{h_{2}}(3.25,0)\right. \\
\left.+\frac{7}{90} W_{h_{2}}(2.5,1 / 3)+\frac{14}{90} W_{h_{2}}(3.25,1 / 3)\right) \mid \\
\leq C\|w\|_{2,2, \infty,[2.5,3.25] \times[0,1 / 3]}\left(h_{2}^{2}+\tau_{2}^{2}\right) .
\end{gathered}
$$

If the exact solution is less smooth near $B_{2}^{h_{1}} \times[0, T]$, then higher accuracy stencils should be applied. However, determining the region where the solution is less smooth would require some estimates (see [24]), for the smoothness of the solution.

2.5. Global discretization. A global discretization of (1.1) can be obtained from the local discretizations (2.2) once the boundary data $g_{h_{l}, B_{2}}^{k}$ are specified using the intergrid interpolation map $\mathcal{I}_{h_{l}}^{k}$.

For each $l=1, \ldots, p$, let $0 \leq \theta_{l} \leq 1$ be the choice of the $\theta$-scheme on $\Omega_{l}^{*} \times$ $[0, T]$. Multiplying each equation in $(2.2)$ by $\tau_{l}$ and rearranging terms, the global discretization of (1.1) becomes

$$
\left\{\begin{aligned}
&\left(I+\tau_{l} \theta_{l} A_{I I}^{h_{l}}\right) U_{h_{l}, I}^{k+1}=\left(I-\tau_{l} \tilde{\theta}_{l} A_{I I}^{h_{l}}\right) U_{h_{l}, I}^{k} \\
&+\tau_{l}\left(-\theta_{l} A_{I B}^{h_{l}} U_{h_{l}, I}^{k+1}+\tilde{\theta}_{l} A_{I B}^{h_{l}} U_{h_{l}, I}^{k}\right) \\
&+\tau_{l}\left(\theta_{l} f_{h_{l}, I}^{k+1}+\tilde{\theta}_{l} f_{h_{l}, I}^{k}\right), \\
& 0, \\
& U_{h_{l}, B_{1}}^{k+1}= \mathcal{I}_{h_{l}}^{k+1}\left(U_{h_{1}, \tau_{1}}, \ldots, U_{h_{p}, \tau_{p}}\right)^{T}, \\
& U_{h_{l}, B_{2}}^{k+1}= \pi_{h_{l}, I} u_{0}, \\
& U_{h_{l}, I}^{0}=
\end{aligned}\right.
$$

for $l=1, \ldots, p$ and $k=0, \ldots, N_{l}-1$.

The above system couples the $p$ local parabolic discretizations through the $U_{h_{l}, B_{2}}^{k+1}=\mathcal{I}_{h_{l}}^{k+1} U_{h, \tau}$ terms. It yields a very large system of linear equations whose parallel iterative solution will be described next. Techniques will also be described for reducing the size of the system and the local memory requirements.

2.6. A parallel Schwarz iterative method. The linear system (2.4) can be solved by a parallel version of the Schwarz iterative method [26 15, 16, 35, 28, 12 which will (under assumptions stated in Section 4) converge geometrically. On a parallel architecture, each processor can in principle be assigned to a different spacetime grid. Some communication between processors will, however, be necessary (to compute the boundary conditions $g_{h_{l}, B_{2}}^{k}$ involving the intergrid interpolation maps $\mathcal{I}_{h_{l}}^{k}$ ). The loads may be well balanced if the subproblems are of comparable size.

System (2.4) will be very large in general, involving all the unknowns on all the space-time grids. However, with some care, the number of unknowns and the memory requirements can be reduced.

1. First, suppose $m$ is a common factor of the number of time steps $N_{l}$ on each grid for $l=1, \ldots, p$ (i.e., $\left.m=\operatorname{gcd}\left(N_{1}, \ldots, N_{p}\right)\right)$. Then, $T$ can be reduced by a factor $m$ by defining $\tilde{T} \equiv T / m$ and repeatedly applying the global scheme on the time intervals $[0, \tilde{T}],[\tilde{T}, 2 \tilde{T}], \ldots,[(m-1) \tilde{T}, m \tilde{T}]$. 
For example, suppose there are two subregions (i.e., $p=2$ ) and the time steps are $\tau_{1}=T / 100$ and $\tau_{2}=T / 200$. Then choose $\tilde{T}=\tau_{1}=2 \tau_{2}$ and repeatedly apply the global discretization scheme $m=100$ times on $\left[0, \tau_{1}\right]$, $\left[\tau_{1}, 2 \tau_{1}\right], \ldots,\left[99 \tau_{1}, 100 \tau_{1}\right]$ to obtain a solution on the time interval $[0, T]$. This procedure will reduce the size of the linear system by a factor 100 .

2. Within each space-time grid $\Omega_{h_{l}}^{*} \times\left\{0, \tau_{1}, \ldots, N_{l} \tau_{l}\right\}$ the discrete solution $U_{h_{l}}^{k}$ need not be stored for $k=0, \ldots, N_{l}$. Store the initial data $U_{h_{l}, I}^{0}$ and the boundary data $g_{h_{l}, B_{2}}^{k}$ for $k=0, \ldots, N_{l}$. Using these, the local discrete solution can be generated by solving the local equations. For the parallel Schwarz algorithm, described next, it would also be necessary to store the nodal values of $\left(U_{h_{l}}^{k}\right)_{i}$ that will be used to compute the intergrid interpolation maps.

Once the size of the global system has been reduced by reducing $N_{l}$ so that $\operatorname{gcd}\left(N_{1}, \ldots, N_{p}\right)=1$, then system (2.4) can be solved by a parallel Schwarz iterative algorithm. To distinguish the different iterates in the Schwarz procedure, we introduce the following notation: $U_{h_{l}}^{k ;(n)}$ will denote the $n$th Schwarz iterate at time $k \tau_{l}$ on the grid $\Omega_{h_{l}}^{*}$.

Parallel Schwarz iteration. Let $\left\{\left\{U_{h_{l}}^{k ;(0)}\right\}_{k=0}^{N_{l}}\right\}_{l=1}^{p}$ be a given starting guess.

1. For $n=0,1, \ldots$ until convergence do

2. For $l=1, \ldots, p$ in parallel do

3. Compute the local boundary conditions for $k=1, \ldots, N_{l}$ :

$$
g_{h_{l}, B_{2}}^{k ;(n+1)}=\mathcal{I}_{h_{l}}^{k}\left(U_{h_{1}, \tau_{1}}^{(n)}, \ldots, U_{h_{p}, \tau_{p}}^{(n)}\right)^{T} .
$$

4. EndFor

5. For $l=1, \ldots, p$ in parallel do

6. Let

$$
U_{h_{l}, I}^{0 ;(n+1)}=\pi_{h_{l}, I} u_{0}
$$

7. For $k=0, \ldots, N_{l}-1$ solve

$$
\begin{aligned}
\left(I+\tau_{l} \theta_{l} A_{I I}^{h_{l}}\right) U_{h_{l}, I}^{k+1 ;(n+1)}= & \left(I-\tau_{l} \tilde{\theta}_{l} A_{I I}^{h_{l}}\right) U_{h_{l}, I}^{k ;(n+1)} \\
& +\tau_{l}\left(-\theta_{l} A_{I B}^{h_{l}} U_{h_{l}, I}^{k+1 ;(n)}+\tilde{\theta}_{l} A_{I B}^{h_{l}} U_{h_{l}, I}^{k ;(n+1)}\right) \\
& +\tau_{l}\left(\theta_{l} f_{h_{l}, I}^{k+1}+\tilde{\theta}_{l} f_{h_{l}, I}^{k}\right) \\
& 0, \\
U_{h_{l}, B_{1}}^{k+1 ;(n+1)}= & \\
U_{h_{l}, B_{2}}^{k+1 ;(n+1)}= & g_{h_{l}, B_{2}}^{k+1 ;(n+1)} .
\end{aligned}
$$

\section{8. $\quad$ EndFor}

9. Endfor

\section{Endfor}

Under suitable assumptions, the iterates $\left\{U_{h_{l}}^{k ;(n)}\right\}$ above can be shown to converge geometrically to the exact solution of (2.4) as $n \rightarrow \infty$ (see Section 4). 


\section{MAXIMUM NORM PROPERTIES OF THE LOCAL DISCRETIZATIONS}

In this section we describe several background results. For local discretizations of parabolic equations, we describe maximum norm stability, maximum principles, comparison theorems, barrier functions, and contraction properties. These results will be used in Section 4 to study the maximum norm stability of the global discretization (2.4). The proofs are presented here for the convenience of the reader, though most of the results are scattered in the literature [34, 14, 25, 21, 28, 10.

3.1. Maximum norm stability and a priori estimates for local discretizations. The following preliminary result provides the basis for maximum norm estimates of solutions to $\theta$-schemes.

Lemma 3.1. Suppose the following hold.

1. Let $A^{h_{l}}=\left[\begin{array}{ll}A_{I I}^{h_{l}} & A_{I B}^{h_{l}}\end{array}\right]$ satisfy Assumption $A 1$.

2. Let $0 \leq \theta_{l} \leq 1$ and let $0<\tau_{l}$.

3. Let $w_{h_{l}}=\left[w_{h_{l}, I}, w_{h_{l}, B}\right]^{T}$ satisfy

$$
\begin{aligned}
\left(I+\tau_{l} \theta_{l} A_{I I}^{h_{l}}\right) w_{h_{l}, I}+\tau_{l} \theta_{l} A_{I B}^{h_{l}} w_{h_{l}, B} & =\tilde{f}_{h_{l}, I} \\
w_{h_{l}, B} & =\tilde{g}_{h_{l}, B} .
\end{aligned}
$$

Then the following holds:

$$
\left|w_{h_{l}}\right|_{\infty} \leq \max \left\{\frac{1}{1+\tau_{l} \theta_{l} c_{0}}\left|\tilde{f}_{h_{l}, I}\right|_{\infty},\left|\tilde{g}_{h_{l}, B}\right|_{\infty}\right\} .
$$

Proof. Without loss of generality (if needed multiply $w_{h_{l}}$ by -1 ), let $\left(w_{h_{l}}\right)_{i}=$ $\left|w_{h_{l}}\right|_{\infty}$ (i.e., $\left(w_{h_{l}}\right)_{i} \geq\left|\left(w_{h_{l}}\right)_{j}\right|$ for all $j$ ). If $i \in B^{h_{l}}$, then since $w_{h_{l}, B}=\tilde{g}_{h_{l}, B}$ the desired result holds. Therefore, in the following, suppose $i \in I^{h_{l}}$. Then the following holds:

$$
\begin{aligned}
\left(\tilde{f}_{h_{l}, I}\right)_{i} & =\left(1+\tau_{l} \theta_{l} A_{i i}^{h_{l}}\right)\left(w_{h_{l}}\right)_{i}+\tau_{l} \theta_{l} \sum_{j \neq i} A_{i j}^{h_{l}}\left(w_{h_{l}}\right)_{j} \\
& =\left(1+\tau_{l} \theta_{l} A_{i i}^{h_{l}}\right)\left|w_{h_{l}}\right|_{\infty}+\tau_{l} \theta_{l} \sum_{j \neq i} A_{i j}^{h_{l}}\left(w_{h_{l}}\right)_{j} \\
& \geq\left(1+\tau_{l} \theta_{l} A_{i i}^{h_{l}}\right)\left|w_{h_{l}}\right|_{\infty}+\tau_{l} \theta_{l} \sum_{j \neq i} A_{i j}^{h_{l}}\left|w_{h_{l}}\right|_{\infty}, \quad \text { since } A_{i j}^{h_{l}} \leq 0 \text { for } i \neq j \\
& =\left(1+\tau_{l} \theta_{l} A_{i i}^{h_{l}}+\sum_{j \neq i} \tau_{l} \theta_{l} A_{i j}^{h_{l}}\right)\left|w_{h_{l}}\right|_{\infty} \\
& =\left(1+\tau_{l} \theta_{l} c_{i}^{h_{l}}\right)\left|w_{h_{l}}\right|_{\infty} \\
& \geq\left(1+\tau_{l} \theta_{l} c_{0}\right)\left|w_{h_{l}}\right|_{\infty} .
\end{aligned}
$$

Thus, $\left|w_{h_{l}}\right|_{\infty} \leq\left|\left(\tilde{f}_{h_{l}, I}\right)_{i}\right| /\left(1+\tau_{l} \theta_{l} c_{0}\right) \leq\left|\tilde{f}_{h_{l}, I}\right|_{\infty} /\left(1+\tau_{l} \theta_{l} c_{0}\right)$.

The preceding result can be applied to the linear system occurring at each time step in the $\theta$-scheme, provided a stability constraint is satisfied by $\tau_{l}$.

Lemma 3.2. Suppose the following hold.

1. Let matrix $A^{h_{l}}$ satisfy Assumption A1.

2. Let $0 \leq \theta_{l} \leq 1$ and define $\tilde{\theta}_{l} \equiv 1-\theta_{l}$. 
3. Let $0<\tau_{l}$ satisfy the stability constraint

$$
\tau_{l} \leq \min _{i \in I^{h_{l}}} \frac{1}{\tilde{\theta}_{l} A_{i i}^{h_{l}}}, \quad \text { if } \tilde{\theta}_{l} \neq 0 .
$$

4. Let $U_{h_{l}}^{k+1}$ satisfy

$$
\begin{aligned}
\left(I+\tau_{l} \theta_{l} A_{I I}^{h_{l}}\right) U_{h_{l}, I}^{k+1}+\tau_{l} \theta_{l} A_{I B}^{h_{l}} U_{h_{l}, B}^{k+1}= & \left(I-\tau_{l} \tilde{\theta}_{l} A_{I I}^{h_{l}}\right) U_{h_{l}, I}^{k}-\tau_{l} \tilde{\theta}_{l} A_{I B}^{h_{l}} U_{h_{l}, B}^{k} \\
& +\tau_{l}\left(\theta_{l} f_{h_{l}, I}^{k+1}+\tilde{\theta}_{l} f_{h_{l}, I}^{k}\right), \\
U_{h_{l}, B}^{k+1}= & g_{h_{l}, B}^{k+1},
\end{aligned}
$$

where $U_{h_{l}, I}^{k}, U_{h_{l}, B}^{k}, g_{h_{l}, B}^{k+1}, f_{h_{l}, I}^{k+1}$ and $f_{h_{l}, I}^{k}$ are given.

Then the following holds:

$$
\begin{aligned}
\left|U_{h_{l}}^{k+1}\right|_{\infty} \leq \max \{ & \left|g_{h_{l}, B}^{k+1}\right|_{\infty}, \frac{\tau_{l} \theta_{l}}{1+\tau_{l} \theta_{l} c_{0}}\left|f_{h_{l}, I}^{k+1}\right|_{\infty} \\
& \left.+\frac{\tau_{l} \tilde{\theta}_{l}}{1+\tau_{l} \theta_{l} c_{0}}\left|f_{h_{l}, I}^{k}\right|_{\infty}+\frac{1-\tau_{l} \tilde{\theta}_{l} c_{0}}{1+\tau_{l} \theta_{l} c_{0}}\left|U_{h_{l}}^{k}\right|_{\infty}\right\} .
\end{aligned}
$$

Proof. We apply the preceding lemma with

$$
\begin{aligned}
\tilde{f}_{h_{l}, I} & \equiv\left(I-\tau_{l} \tilde{\theta}_{l} A_{I I}^{h_{l}}\right) U_{h_{l}, I}^{k}-\tau_{l} \tilde{\theta}_{l} A_{I B}^{h_{l}} U_{h_{l}, B}^{k}+\tau_{l}\left(\theta f_{h_{l}, I}^{k+1}+\tilde{\theta}_{l} f_{h_{l}, I}^{k}\right), \\
\tilde{g}_{h_{l}, B} & \equiv g_{h_{l}, B}^{k+1} .
\end{aligned}
$$

We need to estimate $\left|\tilde{f}_{h_{l}, I}\right|_{\infty}$. Since $1-\tau_{l} \theta_{l} A_{i i} \geq 0$ for all $i$ (by the stability criterion for $\tau_{l}$ ) and $-\tau_{l} \theta_{l} A_{i j}^{h_{l}} \geq 0$ for $i \neq j$, we obtain

$$
\begin{aligned}
\left|\left(\tilde{f}_{h_{l}, I}\right)_{i}\right| \leq & \left(1-\tau_{l} \theta_{l} A_{i i}^{h_{l}}\right)\left|\left(U_{h_{l}}^{k}\right)_{i}\right|-\tau_{l} \theta_{l} \sum_{j \neq i} A_{i j}^{h_{l}}\left|\left(U_{h_{l}}^{k}\right)_{j}\right| \\
& +\tau_{l}\left(\theta_{l}\left|\left(f_{h_{l}, I}^{k+1}\right)_{i}\right|+\tilde{\theta}_{l}\left|\left(f_{h_{l}}^{k}\right)_{i}\right|\right) \\
\leq & \left(1-\tau_{l} \theta_{l} \sum_{j} A_{i j}^{h_{l}}\right)\left|U_{h_{l}}^{k}\right|_{\infty} \\
& +\tau_{l}\left(\theta_{l}\left|f_{h_{l}, I}^{k+1}\right|_{\infty}+\tilde{\theta}_{l}\left|f_{h_{l}, I}^{k}\right|_{\infty}\right) \\
\leq & \left(1-\tau_{l} \theta_{l} c_{0}\right)\left|U_{h_{l}}^{k}\right|_{\infty} \\
& +\tau_{l}\left(\theta_{l}\left|f_{h_{l}, I}^{k+1}\right|_{\infty}+\tilde{\theta}_{l}\left|f_{h_{l}, I}^{k}\right|_{\infty}\right),
\end{aligned}
$$

where we used that $-\tau_{l} \theta_{l} \sum_{j} A_{i j}^{h_{l}} \leq-\tau_{l} \theta_{l} c_{0}$. The desired result now follows by an application of the preceding lemma.

By recursively applying the preceding result for $k=0,1, \ldots, N_{l}-1$, one can obtain an a priori estimate for the solution to the discretized local parabolic equations.

Lemma 3.3. Suppose the following hold.

1. Let matrix $A^{h_{l}}$ satisfy Assumption A1.

2. Let $0 \leq \theta_{l} \leq 1$ and define $\tilde{\theta}_{l} \equiv 1-\theta_{l}$. 
3. Let $0<\tau_{l}$ satisfy the stability constraint

$$
\tau_{l} \leq \min _{i \in I^{h_{l}}} \frac{1}{\tilde{\theta}_{l} A_{i i}^{h_{l}}}, \quad \text { if } \tilde{\theta}_{l} \neq 0 .
$$

4. Let $U_{h_{l}, I}^{k+1}$ satisfy

$$
\begin{aligned}
\left(I+\tau_{l} \theta_{l} A_{I I}^{h_{l}}\right) U_{h_{l}, I}^{k+1}+\tau_{l} \theta_{l} A_{I B}^{h_{l}} U_{h_{l}, B}^{k+1}= & \left(I-\tau_{l} \tilde{\theta}_{l} A_{I I}^{h_{l}}\right) U_{h_{l}, I}^{k}-\tau_{l} \tilde{\theta}_{l} A_{I B}^{h_{l}} U_{h_{l}, B}^{k} \\
& +\tau_{l}\left(\theta_{l} f_{h_{l}, I}^{k+1}+\tilde{\theta}_{l} f_{h_{l}, I}^{k}\right), \\
U_{h_{l}, B}^{k+1}= & g_{h_{l}, B}^{k+1},
\end{aligned}
$$

for $k=0,1, \ldots, N_{l}-1$, where $\left\{g_{h_{l}, B}^{k}\right\}_{k=0}^{N_{l}},\left\{f_{h_{l}, I}^{k}\right\}_{k=0}^{N_{l}}$ and $U_{h_{l}, I}^{0}$ are given.

Then the following holds:

$$
\begin{aligned}
\left|U_{h_{l}}^{N_{l}}\right|_{\infty} \leq & \max \left\{\left|g_{h_{l}, B}^{N_{l}}\right|_{\infty}, \frac{1-\tau_{l} \tilde{\theta}_{l} c_{0}}{1+\tau_{l} \theta_{l} c_{0}}\left|g_{h_{l}, B}^{N_{l}-1}\right|_{\infty}, \ldots,\left(\frac{1-\tau_{l} \tilde{\theta}_{l} c_{0}}{1+\tau_{l} \theta_{l} c_{0}}\right)^{N_{l}}\left|g_{h_{l}, B}^{0}\right|_{\infty}\right\} \\
& +\tau_{l} \sum_{k=0}^{N_{l}-1}\left(\theta_{l}\left(\frac{1-\tau_{l} \tilde{\theta}_{l} c_{0}}{1+\tau_{l} \theta_{l} c_{0}}\right)^{N_{l}-k}\left|f_{h_{l}, I}^{k+1}\right|_{\infty}\right. \\
& \left.+\tilde{\theta}_{l}\left(\frac{1-\tau_{l} \tilde{\theta}_{l} c_{0}}{1+\tau_{l} \theta_{l} c_{0}}\right)^{N_{l}-k}\left|f_{h_{l}, I}^{k}\right|_{\infty}\right) \\
& +\left(\frac{1-\tau_{l} \tilde{\theta}_{l} c_{0}}{1+\tau_{l} \theta_{l} c_{0}}\right)^{N_{l}}\left|U_{h_{l}, I}^{0}\right|_{\infty} .
\end{aligned}
$$

Proof. The proof follows by a recursive application of the preceding lemma.

Remark 3. By setting $c_{0}=0$ in the preceding result, we may obtain a less sharp result:

$$
\begin{aligned}
\left|U_{h_{l}}^{N_{l}}\right|_{\infty} \leq & \max \left\{\left|g_{h_{l}, B}^{N_{l}}\right|_{\infty},\left|g_{h_{l}, B}^{N_{l}-1}\right|_{\infty}, \ldots,\left|g_{h_{l}, B}^{0}\right|_{\infty}\right\} \\
& +\tau \theta\left|f_{h_{l}, I}^{N_{l}}\right|_{\infty}+\tau\left(\sum_{k=1}^{N_{l}-1}\left|f_{h_{l}, I}^{k}\right|_{\infty}\right)+\tau \tilde{\theta}\left|f_{h_{l}, I}^{0}\right|_{\infty} \\
& +\left|U_{h_{l}, I}^{0}\right|_{\infty} .
\end{aligned}
$$

Remark 4. If $f_{h_{l}, I}^{k} \equiv 0$ for $k=0, \ldots, N_{l}$ and $U_{h_{l}, I}^{0}=0$, then the above result yields

$$
\left|U_{h_{l}}^{N_{l}}\right|_{\infty} \leq \max \left\{\left|g_{h_{l}, B}^{N_{l}}\right|_{\infty},\left|g_{h_{l}, B}^{N_{l}-1}\right|_{\infty}, \ldots,\left|g_{h_{l}, B}^{0}\right|_{\infty}\right\},
$$

which is a form of the discrete maximum principle for homogeneous solutions of the local discretized parabolic equation.

3.2. Maximum principles and comparison theorems. The following is a maximum principle for the locally discretized parabolic equations.

Lemma 3.4. Suppose the following hold.

1. Let matrix $A^{h_{l}}$ satisfy Assumption A1.

2. Let $0 \leq \theta_{l} \leq 1$ and define $\tilde{\theta}_{l} \equiv 1-\theta_{l}$. 
3. Let $0<\tau_{l}$ satisfy the stability constraint

$$
\tau_{l} \leq \min _{i \in I^{h_{l}}} \frac{1}{\tilde{\theta}_{l} A_{i i}^{h_{l}}}, \quad \text { if } \tilde{\theta}_{l} \neq 0 .
$$

4. Let $U_{h_{l}, I}^{k+1}$ satisfy

$$
\begin{aligned}
\left(I+\tau_{l} \theta_{l} A_{I I}^{h_{l}}\right) U_{h_{l}, I}^{k+1}+\tau_{l} \theta_{l} A_{I B}^{h_{l}} U_{h_{l}, B}^{k+1}= & \left(I-\tau_{l} \tilde{\theta}_{l} A_{I I}^{h_{l}}\right) U_{h_{l}, I}^{k}-\tau_{l} \tilde{\theta}_{l} A_{I B}^{h_{l}} U_{h_{l}, B}^{k} \\
& +\tau_{l}\left(\theta_{l} f_{h_{l}, I}^{k+1}+\tilde{\theta}_{l} f_{h_{l}, I}^{k}\right), \\
U_{h_{l}, B}^{k+1}= & g_{h_{l}, B}^{k+1},
\end{aligned}
$$

for $k=0,1, \ldots, N_{l}-1$, where $\left\{g_{h_{l}, B}^{k}\right\}_{k=0}^{N_{l}},\left\{f_{h_{l}, I}^{k}\right\}_{k=0}^{N_{l}}$ and $U_{I}^{0}$ are given .

5. Let the initial and boundary data satisfy

$$
\begin{aligned}
\left(U_{h_{l}}^{0}\right)_{i} & \geq 0, \quad i \in I^{h_{l}} \\
\left(f_{h_{l}, I}^{k}\right)_{i} & \geq 0, \quad i \in I^{h_{l}}, \quad k=0, \ldots, N_{l} \\
\left(g_{h_{l}, B}^{k}\right)_{j} & \geq 0, \quad j \in B^{h_{l}}, \quad k=0, \ldots, N_{l} .
\end{aligned}
$$

Then, the following holds:

$$
\left(U_{h_{l}, I}^{k}\right)_{i} \geq 0, \quad i \in I^{h_{l}} \text { and } k=1, \ldots, N_{l} .
$$

Proof. Let $k_{0} \geq 1$ denote the smallest integer such that there is an $i_{0}$ with $\left(U_{h_{l}, I}^{k_{0}}\right)_{i_{0}}<$ 0 (for, if there does not exist such a $k_{0}$ and $i_{0}$, then $\left(U_{h_{l}, I}^{k}\right)_{i} \geq 0$ for all $k$ and $i$, and the desired conclusion holds). Without loss of generality, suppose that

$$
\left(U_{h_{l}, I}^{k_{0}}\right)_{i_{0}}=\min _{i \in I^{h_{l}}}\left(U_{h_{l}, I}^{k_{0}}\right)_{i}<0
$$

Consider the local discretized equation at time $k_{0} \tau_{l}$

$$
\begin{aligned}
\left(I+\tau_{l} \theta_{l} A_{I I}^{h_{l}}\right) U_{h_{l}, I}^{k_{0}}+\tau_{l} \theta_{l} A_{I B}^{h_{l}} U_{h_{l}, B}^{k_{0}}= & \left(I-\tau_{l} \tilde{\theta}_{l} A_{I I}^{h_{l}}\right) U_{h_{l}, I}^{k_{0}-1}-\tau_{l} \tilde{\theta}_{l} A_{I B}^{h_{l}} U_{h_{l}, B}^{k_{0}-1} \\
& +\tau_{l}\left(\theta_{l} f_{h_{l}, I}^{k_{0}}+\tilde{\theta}_{l} f_{h_{l}, I}^{k_{0}-1}\right), \\
U_{h_{l}, B}^{k_{0}}= & g_{h_{l}, B}^{k_{0}} .
\end{aligned}
$$

At the $i_{0}$-th gridpoint this becomes

$$
\begin{aligned}
\left(1+\tau_{l} \theta_{l} A_{i_{0} i_{0}}^{h_{l}}\right)\left(U_{h_{l}}^{k_{0}}\right)_{i_{0}}+\tau_{l} \theta_{l} \sum_{j \neq i_{0}} A_{i_{0} j}^{h_{l}}\left(U_{h_{l}}^{k_{0}}\right)_{j} \\
=\left(I-\tau_{l} \tilde{\theta}_{l} A_{i_{0} i_{0}}^{h_{l}}\right)\left(U_{h_{l}}^{k_{0}-1}\right)_{i_{0}}-\tau_{l} \tilde{\theta}_{l} \sum_{j \neq i_{0}} A_{i_{0} j}^{h_{l}}\left(U_{h_{l}}^{k_{0}-1}\right)_{j} \\
\quad+\tau_{l}\left(\theta_{l}\left(f_{h_{l}, I}^{k_{0}}\right)_{i_{0}}+\tilde{\theta}_{l}\left(f_{h_{l}, I}^{k_{0}-1}\right)_{i_{0}}\right)
\end{aligned}
$$

where $\left(U_{h_{l}, I}^{k_{0}}\right)_{j}=\left(g_{h_{l}, B}^{k}\right)_{j}$ for $j \in B^{h_{l}}$. Using the nonnegativity of $\left(f_{h_{l}, I}^{k_{0}}\right)_{i}$, we obtain

$$
\begin{aligned}
\left(1+\tau_{l} \theta_{l} A_{i_{0} i_{0}}^{h_{l}}\right)\left(U_{h_{l}}^{k_{0}}\right)_{i_{0}}+\tau_{l} \theta_{l} \sum_{j \neq i_{0}} A_{i_{0} j}^{h_{l}}\left(U_{h_{l}}^{k_{0}}\right)_{j} \geq & \left(I-\tau_{l} \tilde{\theta}_{l} A_{i_{0} i_{0}}^{h_{l}}\right)\left(U_{h_{l}}^{k_{0}-1}\right)_{i_{0}} \\
& -\tau_{l} \tilde{\theta}_{l} \sum_{j \neq i_{0}} A_{i_{0} j}^{h_{l}}\left(U_{h_{l}}^{k_{0}-1}\right)_{j} .
\end{aligned}
$$


Since $\left(U_{h_{l}}^{k_{0}-1}\right)_{j} \geq 0$ for all $j,\left(1-\tau_{l} \tilde{\theta}_{l} A_{i_{0} i_{0}}^{h_{l}}\right) \geq 0$ and $-\tau_{l} \tilde{\theta}_{l} \sum_{j \neq i_{0}} A_{i_{0} j}^{h_{l}} \geq 0$, we obtain

$$
\begin{aligned}
\left(1+\tau_{l} \theta_{l} A_{i_{0} i_{0}}^{h_{l}}\right)\left(U_{h_{l}}^{k_{0}}\right)_{i_{0}}+\tau_{l} \theta_{l} \sum_{j \neq i_{0}} A_{i_{0} j}^{h_{l}}\left(U_{h_{l}}^{k_{0}}\right)_{j} \geq & \left(1-\tau_{l} \tilde{\theta}_{l} A_{i_{0} i_{0}}^{h_{l}}\right)\left(U_{h_{l}}^{k_{0}-1}\right)_{i_{0}} \\
& -\tau_{l} \tilde{\theta}_{l} \sum_{j \neq i_{0}} A_{i_{0} j}^{h_{l}}\left(U_{h_{l}}^{k_{0}-1}\right)_{j} \\
\geq & 0 .
\end{aligned}
$$

By assumption $-\left(U_{h_{l}}^{k_{0}}\right)_{i_{0}} \geq-\left(U_{h_{l}}^{k_{0}}\right)_{j}$ and $A_{i_{0} j}^{h_{l}} \leq 0$ for $j \neq i_{0}$. Rearranging terms in the left hand side above, we obtain

$$
\begin{aligned}
0 & \leq\left(1+\tau_{l} \theta_{l} A_{i_{0} i_{0}}^{h_{l}}\right)\left(U_{h_{l}}^{k_{0}}\right)_{i_{0}}+\tau_{l} \theta_{l} \sum_{j \neq i_{0}} A_{i_{0} j}^{h_{l}}\left(U_{h_{l}}^{k_{0}}\right)_{j} \\
& \leq\left(1+\tau_{l} \theta_{l} A_{i_{0} i_{0}}^{h_{l}}\right)\left(U_{h_{l}}^{k_{0}}\right)_{i_{0}}+\tau_{l} \theta_{l} \sum_{j \neq i_{0}} A_{i_{0} j}^{h_{l}}\left(U_{h_{l}}^{k_{0}}\right)_{i_{0}} \\
& =\left(1+\tau_{l} \theta_{l} \sum_{j} A_{i_{0} j}^{h_{l}}\right)\left(U_{h_{l}}^{k_{0}}\right)_{i_{0}} \\
& =\left(1+\tau_{l} \theta_{l} c_{i_{0}}^{h_{l}}\right)\left(U_{h_{l}}^{k_{0}}\right)_{i_{0}} \\
& <0,
\end{aligned}
$$

since $1+\tau_{l} \theta_{l} c_{i_{0}}^{h_{l}} \geq 1$ and $\left(U_{h_{l}}^{k_{0}}\right)_{i_{0}}<0$ (by assumption). We have thus arrived at a contradiction. Therefore, our assumption was incorrect and we must have

$$
\left(U_{h_{l}}^{k}\right)_{i} \geq 0, \quad \text { for } k=0, \ldots, N_{l} \text { and } i \in I^{h_{l}},
$$

which is the desired conclusion.

Remark 5. A similar result can be shown to hold when $c_{0}=0$, provided $A^{h_{l}}$ is an $M$-matrix.

As an immediate application of the preceding lemma, we obtain the following comparison principle.

Lemma 3.5. Suppose the following hold.

1. Let matrix $A^{h_{l}}$ satisfy Assumption A1.

2. Let $0 \leq \theta_{l} \leq 1$ and define $\tilde{\theta}_{l} \equiv 1-\theta_{l}$.

3. Let $0<\tau_{l}$ satisfy the stability constraint

$$
\tau_{l} \leq \min _{i \in I^{h_{l}}} \frac{1}{\tilde{\theta}_{l} A_{i i}^{h_{l}}}, \quad \text { if } \tilde{\theta}_{l} \neq 0 .
$$

4. Let $U_{h_{l}, I}^{k+1}$ satisfy

$$
\begin{aligned}
\left(I+\tau_{l} \theta_{l} A_{I I}^{h_{l}}\right) U_{h_{l}, I}^{k+1}+\tau_{l} \theta_{l} A_{I B}^{h_{l}} U_{h_{l}, B}^{k+1}= & \left(I-\tau_{l} \tilde{\theta}_{l} A_{I I}^{h_{l}}\right) U_{h_{l}, I}^{k}-\tau_{l} \tilde{\theta}_{l} A_{I B}^{h_{l}} U_{h_{l}, B}^{k}, \\
& +\tau_{l}\left(\theta_{l} f_{h_{l}, I}^{k+1}+\tilde{\theta}_{l} f_{h_{l}, I}^{k}\right), \\
U_{h_{l}, B}^{k+1}= & g_{h_{l}, B}^{k+1},
\end{aligned}
$$

for $k=0,1, \ldots, N_{l}-1$, where $\left\{f_{h_{l}, I}^{k}\right\}_{k=0}^{N_{l}},\left\{g_{h_{l}, B}^{k}\right\}_{k=0}^{N_{l}}$ and $U_{h_{l}, I}^{0}$ are given. 
5. Let $\tilde{U}_{I}^{k+1}$ satisfy

$$
\begin{aligned}
\left(I+\tau_{l} \theta_{l} A_{I I}^{h_{l}}\right) \tilde{U}_{h_{l}, I}^{k+1}+\tau \theta A_{I B}^{h_{l}} \tilde{U}_{h_{l}, B}^{k+1}= & \left(I-\tau_{l} \tilde{\theta}_{l} A_{I I}^{h_{l}}\right) \tilde{U}_{h_{l}, I}^{k}-\tau_{l} \tilde{\theta}_{l} A_{I B}^{h_{l}} \tilde{U}_{h_{l}, B}^{k} \\
& +\tau_{l}\left(\theta_{l} \tilde{f}_{h_{l}, I}^{k+1}+\tilde{\theta}_{l} \tilde{f}_{h_{l}, I}^{k}\right) \\
\tilde{U}_{h_{l}, B}^{k+1}= & \tilde{g}_{h_{l}, B}^{k+1},
\end{aligned}
$$

for $k=0,1, \ldots, N_{l}-1$, where $\left\{\tilde{f}_{h_{l}, I}^{k}\right\}_{k=0}^{N_{l}},\left\{\tilde{g}_{h_{l}, B}^{k}\right\}_{k=0}^{N_{l}}$ and $\tilde{U}_{h_{l}, I}^{0}$ are given .

6. Let the forcing terms, the initial and boundary data satisfy:

$$
\begin{aligned}
& \left(U_{h_{l}, I}^{0}\right)_{i} \geq\left(\tilde{U}_{h_{l}, I}^{0}\right)_{i}, \quad i \in I^{h_{l}}, \\
& \left(f_{h_{l}, I}^{k}\right)_{i} \geq\left(\tilde{f}_{h_{l}, I}^{k}\right)_{i}, \quad i \in I^{h_{l}}, \quad k=0, \ldots, N_{l}, \\
& \left(g_{h_{l}, B}^{k}\right)_{j} \geq\left(\tilde{g}_{h_{l}, B}^{k}\right)_{j}, \quad j \in B^{h_{l}}, \quad k=0, \ldots, N_{l} .
\end{aligned}
$$

Then, the following will hold:

$$
\left(U_{h_{l}, I}^{k}\right)_{i} \geq\left(\tilde{U}_{h_{l}, I}^{k}\right)_{i} \quad \forall i \in I^{h_{l}} \text { and } k=1, \ldots, N_{l} .
$$

Proof. Subtract the two sets of discretized parabolic equations to obtain

$$
\begin{aligned}
& \left(I+\tau_{l} \theta_{l} A_{I I}^{h_{l}}\right)\left(U_{h_{l}, I}^{k+1}-\tilde{U}_{h_{l}, I}^{k+1}\right)+\tau_{l} \theta A_{I B}^{h_{l}}\left(U_{h_{l}, B}^{k+1}-\tilde{U}_{h_{l}, B}^{k+1}\right) \\
& =\left(I-\tau_{l} \tilde{\theta}_{l} A_{I I}^{h_{l}}\right)\left(U_{h_{l}, I}^{k}-\tilde{U}_{h_{l}, I}^{k}\right)-\tau_{l} \tilde{\theta}_{l} A_{I B}^{h_{l}}\left(U_{h_{l}, B}^{k}-\tilde{U}_{h_{l}, B}^{k}\right) \\
& +\tau_{l} \theta_{l}\left(f_{h_{l}, I}^{k+1}-\tilde{f}_{h_{l}, I}^{k+1}\right)+\tau_{l} \tilde{\theta}_{l}\left(f_{h_{l}, I}^{k}-\tilde{f}_{h_{l}, I}^{k}\right), \\
& U_{h_{l}, B}^{k+1}-\tilde{U}_{h_{l}, B}^{k+1}=g_{h_{l}, B}^{k+1}-\tilde{g}_{h_{l}, B}^{k+1},
\end{aligned}
$$

for $k=0,1, \ldots, N_{l}-1$ for the differences $\left\{U_{h_{l}, I}^{k}-\tilde{U}_{h_{l}, I}^{k}\right\}$. Since $U_{h_{l}, I}^{0}-\tilde{U}_{h_{l}, I}^{0}$, $g_{h_{l}, B}^{k}-\tilde{g}_{h_{l}, B}^{k}$, and $f_{h_{l}, I}^{k}-\tilde{f}_{h_{l}, I}^{k}$ all have nonnegative entries by assumption, we can apply the preceding maximum principle to obtain

$$
\left(U_{h_{l}, I}^{k}-\tilde{U}_{h_{l}, I}^{k}\right)_{i} \geq 0, \quad \forall i \in I^{h_{l}} \text { for } k=1, \ldots, N_{l},
$$

which is the desired result.

Our primary application of the comparison principle for discretized parabolic equations will be to estimate, on each local grid, the modulus of discrete homogeneous solutions $\tilde{U}_{h_{l}, \tau_{l}}$ with trivial initial data, using a suitable comparison or barrier grid function $w_{h_{l}, \tau_{l}}$. Accordingly, given a discrete homogeneous solution $\tilde{U}_{h_{l}, \tau_{l}}$ satisfying trivial initial conditions $\tilde{U}_{h_{l}}^{0}=0$ and nontrivial boundary conditions $\tilde{U}_{h_{l}, B_{2}}^{k}=\tilde{g}_{h_{l}, B_{2}}^{k}$ for $k=0, \ldots, N_{l}$, we would need to construct (or guarantee the existence of) a suitable grid function $w_{h_{l}, \tau_{l}}$ satisfying the requirements of the preceding lemma. For convenience, we will seek a comparison (or barrier) grid function $w_{h_{l}, \tau_{l}}^{k}$ to be a stationary grid function $w_{h_{l}}$, i.e.,

$$
w_{h_{l}}^{k}=w_{h_{l}}, \quad k=0, \ldots, N_{l}
$$


To ensure applicability of the preceding comparison principle, the requirements on $w_{h_{l}}$ of the preceding lemma will be reduced to

$$
\begin{array}{rlrl}
\left(A_{I I}^{h_{l}} w_{h_{l}, I}+A_{I B}^{h_{l}} w_{h_{l}, B}\right)_{i} & \geq 0, & & i \in I^{h_{l}}, \\
\left(w_{h_{l}, B}\right)_{i} & \geq \max _{k=0, \ldots, N_{l}}\left|\left(\tilde{g}_{h_{l}, B}^{k}\right)_{i}\right|, & i \in B^{h_{l}} \\
\left(w_{h_{l}}\right)_{i} & \geq 0 & i \in I^{h_{l}}
\end{array}
$$

since $\tilde{f}_{h_{l}}^{k}=0$ and $\tilde{U}_{h_{l}}^{0}=0$. We have the following result.

Lemma 3.6. Suppose the following hold.

1. Let matrix $A^{h_{l}}$ satisfy Assumption A1.

2. Let $0 \leq \theta_{l} \leq 1$ and define $\tilde{\theta}_{l} \equiv 1-\theta_{l}$.

3. Let $0<\tau_{l}$ satisfy the stability constraint

$$
\tau_{l} \leq \min _{i \in I^{h_{l}}} \frac{1}{\tilde{\theta}_{l} A_{i i}^{h_{l}}}, \quad \text { if } \tilde{\theta}_{l} \neq 0 .
$$

4. Let $\tilde{U}_{h_{l}, I}^{k+1}$ solve

$$
\begin{aligned}
\left(I+\tau_{l} \theta_{l} A_{I I}^{h_{l}}\right) \tilde{U}_{h_{l}, I}^{k+1}+\tau_{l} \theta_{l} A_{I B}^{h_{l}} \tilde{U}_{h_{l}, B}^{k+1} & =\left(I-\tau_{l} \tilde{\theta}_{l} A_{I I}^{h_{l}}\right) \tilde{U}_{h_{l}, I}^{k}-\tau_{l} \tilde{\theta}_{l} A_{I B}^{h_{l}} \tilde{U}_{h_{l}, B}^{k}, \\
\tilde{U}_{h_{l}, B}^{k+1} & =\tilde{g}_{h_{l}, B}^{k+1},
\end{aligned}
$$

for $k=0,1, \ldots, N_{l}-1$ with $\tilde{U}_{h_{l}, I}^{0}=0$, where $\left\{\tilde{g}_{h_{l}, B}^{k}\right\}_{k=0}^{N_{l}}$ are given.

5. Let $w_{h_{l}}=\left(w_{h_{l}, I}, w_{h_{l}, B}\right)^{T}$ satisfy

$$
\begin{array}{rlrl}
\left(A_{I I}^{h_{l}} w_{h_{l}, I}+A_{I B}^{h_{l}} w_{h_{l}, B}\right)_{i} & \geq 0, & i \in I^{h_{l}}, \\
\left(w_{h_{l}, B}\right)_{j} & \geq \max _{k=0, \ldots, N_{l}}\left|\left(\tilde{g}_{h_{l}, B}\right)_{j}\right|, \quad j \in B^{h_{l}} .
\end{array}
$$

Then, the following holds:

$$
\left(w_{h_{l}, I}\right)_{i} \geq\left|\left(\tilde{U}_{h_{l}, I}^{k}\right)_{i}\right|, \quad \forall i \in I^{h_{l}}, \quad \text { for } k=0, \ldots, N_{l} .
$$

Proof. We will apply the comparison principle (Lemma 3.5) using $\tilde{U}_{h_{l}, \tau_{l}}$ as defined above and

$$
U_{h_{l}}^{k} \equiv w_{h_{l}}, \quad \text { for } k=0, \ldots, N_{l} .
$$

Due to the stationarity of $U_{h_{l}}^{k}$, the finite difference approximation of its time derivative is zero and

$$
\begin{aligned}
\left(I+\tau_{l} \theta_{l} A_{I I}^{h_{l}}\right) & U_{h_{l}, I}^{k+1}+\tau_{l} \theta_{l} A_{I B}^{h_{l}} U_{h_{l}, B}^{k+1} \\
& \geq\left(I-\tau_{l} \tilde{\theta}_{l} A_{I I}^{h_{l}}\right) U_{h_{l}, I}^{k}-\tau_{l} \tilde{\theta}_{l} A_{I B}^{h_{l}} U_{h_{l}, B}^{k}+\tau_{l} \tilde{f}_{h_{l}, I}, \\
\left(U_{h_{l}, B}^{k+1}\right)_{j} & \geq\left|\left(\tilde{g}_{h_{l}, B}^{k+1}\right)_{j}\right|, \quad j \in B^{h_{l}},
\end{aligned}
$$

holds for $k=0, \ldots, N_{l}-1$.

In order to apply Lemma 3.5. we also need to verify that

$$
\left(U_{h_{l}, I}^{0}\right)_{i} \geq\left(\tilde{U}_{h_{l}}^{0}\right)_{i}=0, \quad \forall i \in I^{h_{l}},
$$


which in our application corresponds to $\left(w_{h_{l}, I}\right)_{i} \geq 0$ for all $i$. From the definition of $w_{h_{l}}$, we obtain

$$
w_{h_{l}, I}=\left(A_{I I}^{h_{l}}\right)^{-1}\left(\tilde{f}_{h_{l}, I}-A_{I B}^{h_{l}} w_{h_{l}, B}\right) .
$$

Since $A^{h_{l}}$ satisfies Assumption $A 1$, it is a strictly diagonally dominant (rectangular) $M$-matrix and satisfies

$$
\left(\left(A_{I I}^{h_{l}}\right)^{-1}\right)_{i j} \geq 0, \quad \forall i, j \in I^{h_{l}} .
$$

Since $\left(A^{h_{l}}\right)_{i j} \leq 0$ for $i \neq j, \tilde{f}_{h_{l}}^{k}=0$ and $\left(w_{h_{l}, B}\right)_{i} \geq 0$ in the above, we conclude that $\left(w_{h_{l}, I}\right)_{i} \geq 0$ for $i \in I^{h_{l}}$. By the comparison principle

$$
\left(U_{h_{l}}^{k}\right)_{i}=\left(w_{h_{l}}\right)_{i} \geq\left(\tilde{U}_{h_{l}}^{k}\right)_{i}, \quad \forall i, k .
$$

To complete the proof, we observe that all of the above inequalities remain valid if $\tilde{U}_{h_{l}, I}^{k}$ is replaced by $-\tilde{U}_{h_{l}, I}^{k}$. Applying identical arguments yields

$$
\left(w_{h_{l}}\right)_{i} \geq-\left(\tilde{U}_{h_{l}}^{k}\right)_{i}, \quad \forall i, k .
$$

Combining these two results yields

$$
\left(w_{h_{l}}\right)_{i} \geq\left|\tilde{U}_{h_{l}}^{k}\right|_{i}, \quad \forall i, k,
$$

which is the desired result.

In the next section, we describe a technique for constructing grid functions $w_{h_{l}}$, for sufficiently small mesh size $h_{l}$ (with $h_{l} \leq h_{l}^{*}$ ) when $c_{0}>0$. Such grid functions (actually, standardized versions of them where the boundary values are suitably scaled) will be referred to as discrete barrier or comparison functions. Once barrier functions are constructed (or their properties are known), they can be applied to derive a contraction property for homogeneous solutions.

3.3. Existence of continuous and discrete barrier functions. In this section we prove the existence of a discrete barrier grid function $w_{h_{l}}$ when $c_{0}>0$ on each local grid and describe some of its properties. We state below the precise requirements that a grid function must satisfy in order to be called a barrier function.

Definition. A grid function $w_{h_{l}}=\left(w_{h_{l}, I}, w_{h_{l}, B}\right)^{T}$ that satisfies

$$
\begin{aligned}
\left(A_{I I}^{h_{l}} w_{h_{l}, I}+A_{I B}^{h_{l}} w_{h_{l}, B}\right)_{i} & \geq 0, \quad i \in I^{h_{l}}, \\
\left(w_{h_{l}, B_{1}}\right)_{i} & \geq 0, \quad i \in B_{1}^{h_{l}}, \\
\left(w_{h_{l}, B_{1}}\right)_{i} & \geq 1, \quad i \in B_{2}^{h_{l}},
\end{aligned}
$$

will be referred to as a discrete barrier (or comparison) grid function.

The existence of discrete barrier grid functions will be proved in two stages. First, results on the existence of a continuous barrier function $w_{l}(x)$ associated with the continuous analog of (3.1) on $\Omega_{l}^{*}$ will be described. Second, a grid function $w_{h_{l}}=\pi_{h_{l}} w_{l}$ will be defined by nodal interpolation of $w_{l}(x)$ onto the grid $\Omega_{h_{l}}^{*}$. For sufficiently small $h_{l}$ (i.e., for $h_{l} \leq h_{l}^{*}$ for some $h_{l}^{*}>0$ ), it will be shown that the resulting grid function $w_{h_{l}}$ will satisfy the requirements (3.1). We have the following result for continuous barrier functions. 
Lemma 3.7. Suppose the following hold.

1. Let $0 \leq d_{l}(x)$ denote the distance from $x$ to the boundary segment $B_{2}^{l}=$ $\partial \Omega_{l}^{*} \cap \Omega$ :

$$
d_{l}(x) \equiv \operatorname{dist}\left(x, B_{2}^{l}\right) .
$$

2. Let $w_{l}(x)$ be defined as

$$
w_{l}(x) \equiv e^{-\alpha d_{l}(x)}, \quad x \in \bar{\Omega}_{l}^{*},
$$

for $\alpha>0$.

Then there exists a choice $\alpha=\alpha_{l}>0$ so that $w_{l}(x)$ defined above satisfies

$$
\begin{aligned}
L w_{l} & \geq \frac{c_{0}}{2}, \quad x \in \Omega_{l}^{*}, \\
w_{l}(x) & \geq 0, \quad x \in B_{1}^{l}, \\
w_{l}(x) & =1, \quad x \in B_{2}^{l} .
\end{aligned}
$$

Proof. We follow the construction in Lions 27] (see also [28). Direct computation of $L e^{-\alpha d_{l}(x)}$ yields

$$
\begin{aligned}
L e^{-\alpha d_{l}(x)} & =e^{-\alpha d_{l}(x)}\left(a \alpha \Delta d_{l}(x)-a \alpha^{2}\left|\nabla d_{l}(x)\right|^{2}-\alpha \vec{b}(x) \cdot \nabla d_{l}(x)+c(x)\right) \\
& \geq e^{-\alpha d_{l}(x)}\left(a \alpha \Delta d_{l}(x)-a \alpha^{2}\left|\nabla d_{l}(x)\right|^{2}-\alpha \vec{b}(x) \cdot \nabla d_{l}(x)+c_{0}\right) .
\end{aligned}
$$

If we choose $\alpha=\alpha_{l}>0$

$$
\alpha_{l} \equiv \min \left\{1, \frac{c_{0}}{2\left(\|\vec{b}\|_{\infty, \bar{\Omega}_{l}^{*}}\left\|\nabla d_{l}\right\|_{\infty, \bar{\Omega}_{l}^{*}}+a\left\|\nabla d_{l}\right\|_{\infty, \bar{\Omega}_{l}^{*}}^{2}+a\left\|\Delta d_{l}\right\|_{\infty, \bar{\Omega}_{l}^{*}}\right)}\right\},
$$

then

$$
\frac{c_{0}}{2}+a \alpha \Delta d_{l}(x)-a \alpha^{2}\left|\nabla d_{l}(x)\right|^{2}-\alpha \vec{b}(x) \cdot \nabla d_{l}(x) \geq 0,
$$

and we obtain $L e^{-\alpha_{l} d_{l}(x)} \geq c_{0} / 2>0$. Since $d_{l}(x)=0$ on $B_{2}^{l}$, it follows that $e^{-\alpha d_{l}(x)}=1$ on $B^{l_{2}}$. Since the exponential $e^{-\alpha d_{l}(x)}$ is always nonnegative, it follows that $0 \leq e^{-\alpha d_{l}(x)}$ on $B_{1}^{l}$. Additionally, since $0 \leq d_{l}(x)$ for all $x$, it follows that $0 \leq e^{-\alpha_{l} d_{l}(x)} \leq 1$.

Remark 6. In the above construction, we tacitly assumed smoothness of the distance function $d_{l}(x)$. Unfortunately, this may not be the case in general, even if $B_{2}^{l}=\left(\partial \Omega_{l}^{*} \cap \Omega\right)$ is smooth. However, given any $0<\epsilon_{l} \ll \beta_{l}$, for our applications we may replace $d_{l}(x)$ by any smooth function $0 \leq \tilde{d}_{l ; \epsilon_{l}}(x)$ satisfying

$$
\begin{array}{lll}
\tilde{d}_{l ; \epsilon_{l}}(x) \geq 0, & & \forall x, \\
\tilde{d}_{l ; \epsilon_{l}}(x)=0, & & x \in B_{2}^{l}, \\
\tilde{d}_{l ; \epsilon_{l}}(x) \leq d_{l}(x)+\epsilon_{l}, & \forall x, \\
\tilde{d}_{l ; \epsilon_{l}}(x) \geq d_{l}(x)-\epsilon_{l}, & \forall x .
\end{array}
$$

Such a "pseudo-distance" function $\tilde{d}_{l ; \epsilon_{l}}(x)$ can be constructed as follows. For any $\gamma>0$, let $\Omega_{l}^{\gamma}$ denote

Then $\Omega_{l}^{*}=\Omega_{l}^{\beta_{l}}$.

$$
\Omega_{l}^{\gamma} \equiv\left\{x: \operatorname{dist}\left(x, \Omega_{l}\right)<\gamma\right\}
$$


1. Let $S_{l ; \epsilon_{l}}$ denote a region with smooth boundaries satisfying

$$
\left(\Omega_{l}^{\beta_{l}+\frac{\epsilon_{l}}{2}} \backslash \Omega_{l}^{\beta_{l}-\frac{\epsilon_{l}}{2}}\right) \subset S_{l, \epsilon_{l}} \subset\left(\Omega_{l}^{\beta_{l}+\epsilon_{l}} \backslash \Omega_{l}^{\beta_{l}-\epsilon_{l}}\right) .
$$

Then $B_{2}^{l} \subset S_{l, \epsilon_{l}}$.

2. Given $S_{l, \epsilon_{l}}$, let $d_{l ; \epsilon_{l}}(x)$ denote

$$
d_{l ; \epsilon_{l}}(x) \equiv \operatorname{dist}\left(x, S_{l, \epsilon_{l}}\right) .
$$

Then $d_{l ; \epsilon_{l}}(x)$ will have the following properties:

$$
\begin{array}{lll}
d_{l ; \epsilon_{l}}(x) \geq 0, & & \forall x, \\
d_{l ; \epsilon_{l}}(x)=0, & x \in S_{l ; \epsilon}, \\
d_{l ; \epsilon_{l}}(x) \leq d_{l}(x)+\frac{\epsilon_{l}}{2}, & \forall x, \\
d_{l ; \epsilon_{l}}(x) \geq d_{l}(x)-\frac{\epsilon_{l}}{2}, & \forall x .
\end{array}
$$

Unfortunately, $d_{l ; \epsilon_{l}}(x)$ will not be smooth in the regions where the level sets of $S_{l, \epsilon_{l}}$ intersect.

3. Let $0 \leq \psi_{\epsilon_{l}}(x)$ denote a smooth probability density function having compact support of diameter $\epsilon_{l} / 4$ centered at the origin. Define $\tilde{d}_{l}(x)$ as the convolution (mollification) of $d_{l ; \epsilon_{l}}(x)$ with $\psi_{\epsilon_{l}}(x)$ as

$$
\tilde{d}_{l}(x) \equiv \int_{y} \psi_{\epsilon_{l}}(y) d_{l ; \epsilon_{l}}(x-y) d y .
$$

By construction $\tilde{d}_{l}(x)$ will be smooth. Due to the nonnegativity and compact support of $\psi_{\epsilon_{l}}(x)$ of diameter $\epsilon_{l} / 4$, it will further satisfy

$$
\begin{array}{lrr}
\tilde{d}_{l}(x) & \geq & 0, \quad \forall x, \\
\tilde{d}_{l}(x) & = & 0, \quad x \in B_{2}^{l}, \\
\tilde{d}_{l}(x) \leq & d_{l}(x)+\epsilon_{l}, \quad \forall x, \\
\tilde{d}_{l}(x) \geq & d_{l}(x)-\epsilon_{l}, \quad \forall x .
\end{array}
$$

Thus, given a suitable small but fixed choice of $\epsilon_{l}, e^{-\alpha \tilde{d}_{l}(x)} \leq e^{-\alpha\left(d_{l}(x)+\epsilon_{l}\right)}$ will satisfy the requirements of a barrier function for the value of $\alpha_{l}$ given in the preceding lemma (with $\tilde{d}_{l}(x)$ replacing $d_{l}(x)$ ). For convenience, however, we will henceforth assume that $d_{l}(x)$ is smooth.

Given the continuous barrier function $w_{l}(x)$, we will interpolate it onto the grid $\Omega_{h_{l}}^{*}$ to construct a discrete barrier function. To ensure that the resulting grid function satisfies (3.1), we will require that the discretization $A^{h_{l}}$ be at least first order accurate and that $h_{l}$ be sufficiently small.

Assumption A5. Let $x_{i}^{h_{l}}$ denote an interior gridpoint in $\Omega_{h_{l}}^{*}$. Then for any sufficiently smooth test function $v(x)$ we assume that

$$
(L v)\left(x_{i}^{h_{l}}\right)=\left(A^{h_{l}} \pi_{h_{l}} v\right)_{i}+\mathcal{C}_{h_{l}}\left(v, x_{i}^{h_{l}}\right) h_{l}^{s_{l}}
$$

holds, where the coefficient $\mathcal{C}_{h_{l}}\left(v, x_{i}^{h_{l}}\right)$ involves higher order derivatives of $v(x)$ in the convex hull of the gridpoints of the local stencil

$$
\left|\mathcal{C}_{h_{l}}\left(v, x_{i}^{h_{l}}\right)\right| \leq C\|v\|_{s_{l}+2, \infty, \Omega_{l}^{*}},
$$

where $C$ is a positive constant independent of $h_{l}$ and $v($.$) and 1 \leq s_{l}$ is an integer. 
Lemma 3.8. Suppose the following hold.

1. Let matrix $A^{h_{l}}$ satisfy Assumptions $A 1$ and $A 5$.

2. Let $w_{l}(x)=e^{-\alpha_{l} d_{l}(x)}$ be a smooth continuous barrier function satisfying

$$
\begin{aligned}
& L w_{l} \geq \frac{c_{0}}{2}, \quad x \in \Omega_{l}^{*}, \\
& w_{l}(x) \geq 0, \quad x \in B_{1}^{l}, \\
& w_{l}(x)=1, \quad x \in B_{2}^{l} .
\end{aligned}
$$

3. Define $w_{h_{l}} \equiv \pi_{h_{l}} w_{l}$ where

$$
\left(w_{h_{l}}\right)_{i}=w_{l}\left(x_{i}^{h_{l}}\right), \quad \forall x_{i}^{h_{l}} \in \bar{\Omega}_{h_{l}}^{*} .
$$

4. Let $h_{l}$ satisfy

$$
h_{l} \leq h_{l}^{*} \equiv\left(\frac{c_{0}}{2 C\left\|w_{l}\right\|_{s_{l}+2, \infty, \Omega_{l}^{*}}}\right)^{1 / r_{l}}
$$

where $C$ is defined in (3.2).

Then, the following will hold:

$$
\begin{aligned}
A_{I I}^{h_{l}} w_{h_{l}, I}+A^{h_{l}} w_{h_{l}, B} & \geq 0, \quad \text { componentwise } \\
w_{h_{l}, B_{2}} & \geq 1, \quad \text { componentwise } \\
w_{h_{l}, B_{1}} & \geq 0, \quad \text { componentwise }
\end{aligned}
$$

i.e., $w_{h_{l}}$ is a discrete barrier function.

Proof. By Assumption $A 5$ on the local consistency of the finite difference discretization matrix $A^{h_{l}}$, we obtain

$$
\begin{aligned}
\left(A^{h_{l}} w_{h_{l}}\right)_{i} & =\left(L w_{l}\right)\left(x_{i}^{h_{l}}\right)+\mathcal{C}_{h_{l}}\left(w_{l}, x_{i}^{h_{l}}\right) h_{l}^{s_{l}}, & & \\
& \geq \frac{c_{0}}{2}+\mathcal{C}_{h_{l}}\left(w_{l}, x_{i}^{h_{l}}\right) h_{l}^{s_{l}}, & & \text { since } L w_{l} \geq \frac{c_{0}}{2} \\
& \geq \frac{c_{0}}{2}-C\left\|w_{l}\right\|_{s_{l}+2, \infty, \Omega_{l}^{*}} h_{l}^{s_{l}}, & & \text { using } A 5 \\
& \geq 0, & & \text { if } h_{l} \leq h_{l}^{*},
\end{aligned}
$$

where

$$
h_{l}^{*}=\left(\frac{c_{0}}{2 C\left\|w_{l}\right\|_{s_{l}+2, \infty, \Omega_{l}^{*}}}\right)^{1 / s_{l}} .
$$

The desired result now holds due to the properties of $w_{l}(x)$ and since $w_{h_{l}}=\pi_{h_{l}} w_{l}$.

In the next section, we use barrier grid functions to prove a contraction property of homogeneous solutions to discretized parabolic equations. 
3.4. Contraction property of homogeneous solutions. Suppose $\left\{W_{h_{l}}^{k}\right\}_{k=0}^{N_{l}}$ denotes a homogeneous solution of the discretized parabolic equation on the $l$ th local grid with trivial initial conditions $W_{h_{l}, I}^{0}=0$ and nontrivial boundary data $W_{h_{l}, B_{2}}^{k}=g_{h_{l}, B_{2}}^{k}$ on $B_{2}^{h_{l}}$. By the discrete maximum principle for parabolic equations, we obtain the bound

$$
\left|\left(W_{h_{l}}^{k}\right)_{j}\right| \leq \max _{k=0, \ldots, N_{l}} \max _{\left\{i \in B_{2}^{h_{l}}\right\}}\left|\left(g_{h_{l}, B_{2}}^{k}\right)_{i}\right|,
$$

at any interior gridpoint $x_{j}^{h_{l}}$ at time $k \tau_{l}$ for $k=0, \ldots, N_{l}$. However, when $c_{0}>0$ (as we have assumed), a stronger property will hold in the interior region $\bar{\Omega}_{l} \times[0, T]$

$$
\max _{j \in \bar{\Omega}_{h_{l}}}\left|\left(W_{h_{l}}^{k}\right)_{j}\right| \leq \rho_{h_{l}} \max _{k=0, \ldots, N_{l}} \max _{i \in B_{2}^{h_{l}}}\left|\left(g_{h_{l}, B_{2}}^{k}\right)_{i}\right|,
$$

for some $\rho_{h_{l}}<1$. This will be referred to as the local contraction property and will be essential in establishing the stability of the global discretization.

We define now the normalized contraction factor $0 \leq \rho_{h_{l}} \leq 1$ from a domain $\Omega_{l}^{*}$ to a subregion $\Omega_{l}$ for discrete homogeneous solutions of the discretized parabolic equation on the $l$ th grid.

Definition. Let $\left\{W_{h_{l}}^{k}\right\}_{k=0}^{N_{l}}$ denote a homogeneous solution of the following discretized parabolic equation with trivial initial conditions:

$$
\begin{aligned}
\left(I+\tau_{l} \theta_{l} A_{I I}^{h_{l}}\right) W_{h_{l}, I}^{k+1}+\tau_{l} \theta_{l} A_{I B}^{h_{l}} W_{h_{l}, B}^{k+1} & =\left(I-\tau_{l} \tilde{\theta}_{l} A_{I I}^{h_{l}}\right) W_{h_{l}, I}^{k}-\tau_{l} \tilde{\theta}_{l} A_{I B}^{h_{l}} W_{h_{l}, B}^{k}, \\
W_{h_{l}, B_{1}}^{k+1} & =0 \\
W_{h_{l}, B_{2}}^{k+1} & =g_{h_{l}, B_{2}}^{k}, \\
W_{h_{l}, I}^{0} & =0,
\end{aligned}
$$

where $\left(g_{h_{l}, B_{2}}^{k}\right)_{i}=1$ for $i \in B_{2}^{h_{l}}$. We define the normalized contraction factor $\rho_{h_{l}}$ (with $0 \leq \rho_{h_{l}} \leq 1$ ) as

$$
\rho_{h_{l}} \equiv \max _{k=0, \ldots, N_{l}} \max _{i \in \bar{\Omega}_{h_{l}}}\left|\left(W_{h_{l}, I}^{k}\right)_{i}\right| .
$$

Our first result in this section provides an upper bound for $\rho_{h_{l}}$, in terms of the contraction factor for the continuous barrier function $e^{-\alpha_{l} \tilde{d}_{l}(x)}$ from the preceding section.

Lemma 3.9. Suppose the following hold.

1. Let $0<\beta_{l}$ denote the overlap parameter from subdomain $\Omega_{l}$ to $\Omega_{l}^{*}$.

2. Let matrix $A^{h_{l}}$ satisfy Assumptions $A 1$ and $A 5$, and let $\tau_{l}$ satisfy the stability criterion.

3. Let $c_{0}>0$.

4. Let $0<\alpha_{l}$ be chosen so that the grid function $w_{h_{l}}=\pi_{h_{l}} e^{-\alpha_{l} \tilde{d}_{l}(x)}$ is a discrete barrier grid function for $h_{l} \leq h_{l}^{*}$.

5. Let $\rho_{h_{l}}$ denote the normalized contraction factor on the lth grid with $W_{h_{l}}$ as employed in (3.3). 
Then the following holds:

$$
\begin{aligned}
\rho_{h_{l}} & \leq \max _{i \in \bar{\Omega}_{h_{l}}}\left(w_{h_{l}}\right)_{i} \\
& \leq \max _{i \in \bar{\Omega}_{h_{l}}} e^{-\alpha_{l} \tilde{d}_{l}\left(x_{i}^{h_{l}}\right)} .
\end{aligned}
$$

Consequently

$$
\begin{aligned}
\rho_{h_{l}} & \leq e^{-\alpha_{l} \beta_{l}}, \quad \text { if } \tilde{d}_{l}(x)=d_{l}(x) \\
& \leq e^{-\alpha_{l}\left(\beta_{l}-\epsilon_{l}\right)}, \quad \text { if } d_{l}(x)-\epsilon \leq \tilde{d}_{l}(x) \leq d_{l}(x),
\end{aligned}
$$

for some $\epsilon_{l}>0$. In either case, $\rho_{h_{l}}<1$ if $\beta_{l} \geq \epsilon_{l}$.

Proof. By assumption on the mesh size $h_{l}$, the preceding lemma yields that $w_{h_{l}} \equiv$ $\pi_{h_{l}} e^{-\alpha_{l} \tilde{d}_{l}(x)}$ is a discrete barrier grid function. By applying comparison principle 3.5 from subsection 3.2 , with $U_{h_{l}}^{k}=w_{h_{l}}$ and $\tilde{U}_{h_{l}}^{k}=W_{h_{l}}^{k}$ above, we obtain

$$
\begin{aligned}
\left|\left(W_{h_{l}}^{k}\right)_{i}\right| & \leq\left(w_{h_{l}}\right)_{i} \quad \forall k \\
& \leq e^{-\alpha_{l} \tilde{d}_{l}\left(x_{i}^{h_{l}}\right)} \quad \forall k \\
& \leq e^{-\alpha_{l}\left(\beta_{l}-\epsilon_{l}\right)} \quad \forall k,
\end{aligned}
$$

which is the desired result.

The above estimates for the contraction factor $\rho_{h_{l}}$ are qualitative, and involve an unknown constant $\alpha_{l}$. We indicate below how more quantitative theoretical bounds can be obtained for $\rho_{h_{l}}$ on uniform grids (see [21]).

Example. Consider a parabolic equation $u_{t}+L u=f$ in one space dimension (i.e., $\Omega \subset R$ ) where $L u=-u^{\prime \prime}+b u^{\prime}+c u$ and where $0<b$ and $0<c$ are constants. For convenience, consider a subdomain $\Omega_{l}^{*}=(0,1)$ with $\Omega_{l}=(\tilde{a}, \tilde{b})$ where $0<\tilde{a}<\tilde{b}<$ 1. Suppose that a uniform grid is constructed on $\Omega_{l}^{*}$ with mesh size $h_{l}=1 / M_{l}$ and gridpoints $x_{i}^{h_{l}}=i h_{l}$ for $0, \ldots, M_{l}$. Discretize $-u^{\prime \prime}$ by three point finite differences, $b u^{\prime}$ by upwind finite differences and $c u$ by a one point approximation on the above uniform grid and suppose 0 and 1 are interior points in $\Omega$. To estimate the discrete contraction factor $\rho_{h_{l}}$, we solve the difference equations

$$
\left\{\begin{aligned}
-(b h+1) u_{i-1}+\left(2+b h+c h^{2}\right) u_{i}-1 u_{i+1} & =0, \quad \text { for } i=1, \ldots, M_{l}-1, \\
u_{0} & =1, \\
u_{M_{l}} & =1,
\end{aligned}\right.
$$

whose general solution has the form

$$
u_{i}=c_{1} \sigma_{1}^{i}+c_{2} \sigma_{2}^{i},
$$

where $\sigma_{1}$ and $\sigma_{2}$ are roots of the quadratic

$$
\sigma^{2}-\left(2+b h+c h^{2}\right) \sigma+(b h+1)=0 .
$$


In terms of $b, c$ and $h_{l}$ the roots are

$$
\begin{aligned}
& \sigma_{1}=1+\frac{b h}{2}+\frac{c h^{2}}{2}+h\left(\frac{4 c+b^{2}+2 b h c+c^{2} h^{2}}{4}\right)^{1 / 2}, \\
& \sigma_{2}=1+\frac{b h}{2}+\frac{c h^{2}}{2}-h\left(\frac{4 c+b^{2}+2 b h c+c^{2} h^{2}}{4}\right)^{1 / 2} .
\end{aligned}
$$

The constants $c_{1}$ and $c_{2}$ can be computed by enforcing the boundary conditions

$$
\begin{aligned}
c_{1}+c_{2} & =1, \\
\sigma_{1}^{M_{l}} c_{1}+\sigma_{2}^{M_{l}} c_{2} & =1,
\end{aligned}
$$

which yields

$$
\begin{aligned}
& c_{1}=\frac{\sigma_{2}^{M_{l}}-1}{\sigma_{2}^{M_{l}}-\sigma_{1}^{M_{l}}}, \\
& c_{2}=\frac{1-\sigma_{1}^{M_{l}}}{\sigma_{2}^{M_{l}}-\sigma_{1}^{M_{l}}} .
\end{aligned}
$$

The contraction factor $\rho_{h_{l}}$ can be estimated as

$$
\rho_{h_{l}} \equiv \max _{\left\{i: x_{i}^{h_{l}} \in(\tilde{a}, \tilde{b})\right\}}\left|\frac{\left(\sigma_{2}^{M_{l}}-1\right) \sigma_{1}^{i}+\left(1-\sigma_{1}^{M_{l}}\right) \sigma_{2}^{i}}{\sigma_{2}^{M_{l}}-\sigma_{1}^{M_{l}}}\right| .
$$

Given $b, c, h_{l}, \tilde{a}$ and $\tilde{b}$, these can be determined quantitatively.

Our main result in this section relates the discrete contraction factor $\rho_{h_{l}}$, which was defined for the normalized Dirichlet boundary conditions, to the case of general Dirichlet boundary conditions.

Lemma 3.10. Suppose the following hold.

1. Let matrix $A^{h_{l}}$ satisfy Assumptions $A 1$ and $A 5$.

2. Let $0 \leq \theta_{l} \leq 1$ and define $\tilde{\theta}_{l} \equiv 1-\theta_{l}$.

3. Let $0<\tau_{l}$ satisfy the stability condition

$$
\tau_{l} \leq \min _{i \in I^{h_{l}}} \frac{1}{\tilde{\theta}_{l} A_{i i}^{h_{l}}}, \quad \text { if } \tilde{\theta}_{l} \neq 0 .
$$

4. Let $\tilde{V}_{h_{l}}^{k}$ satisfy

$$
\begin{aligned}
\left(I+\tau_{l} \theta_{l} A_{I I}^{h_{l}}\right) \tilde{V}_{h_{l}, I}^{k+1}+\tau_{l} \theta_{l} A_{I B}^{h_{l}} \tilde{V}_{h_{l}, B}^{k+1} & =\left(I-\tau_{l} \tilde{\theta}_{l} A_{I I}^{h_{l}}\right) \tilde{V}_{h_{l}, I}^{k}-\tau_{l} \tilde{\theta}_{l} A_{I B}^{h_{l}} \tilde{V}_{h_{l}, B}^{k}, \\
\tilde{V}_{h_{l}, B_{1}}^{k+1} & =0, \\
\tilde{V}_{h_{l}, B_{2}}^{k+1} & =\tilde{g}_{h_{l}, B_{2}}^{k+1}, \\
\tilde{V}_{h_{l}, I}^{0} & =0,
\end{aligned}
$$

for $k=0, \ldots, N_{l}-1$.

5. Let $\rho_{h_{l}}$ denote the normalized contraction factor defined by (3.3) in subsection 3.4 with associated grid function $\left\{W_{h_{l}}^{k}\right\}_{k=0}^{N_{l}}$. 
Then the following holds:

$$
\max _{\left\{i: x_{i}^{h_{l}} \in \bar{\Omega}_{h_{l}}, k=0, \ldots, N_{l}\right\}}\left|\left(\tilde{V}_{h_{l}}^{k}\right)_{i}\right| \leq \rho_{h_{l}} \max _{i \in \bar{\Omega}_{h_{l}}^{*}} \max _{k=0, \ldots, N_{l}}\left|\left(\tilde{V}_{h_{l}}^{k}\right)_{i}\right| .
$$

Proof. Let ||$\tilde{V}_{B_{2}^{h_{l}}} \| \mid$ denote the number

$$
||\left|\tilde{V}_{B_{2}^{h_{l}}} \|\right| \equiv \max _{i \in B_{2}^{h_{l}}} \max _{k=0, \ldots, N_{l}}\left|\left(\tilde{V}_{h_{l}}^{k}\right)_{i}\right| .
$$

Define a grid function $\tilde{W}_{h_{l}}^{k}$ by scaling $\tilde{V}_{h_{l}}^{k}$ so that its boundary values have maximum modulus one:

$$
\left(\tilde{W}_{h_{l}}^{k}\right)_{i} \equiv \frac{\left(\tilde{V}_{h_{l}}^{k}\right)_{i}}{\left|\left\|\tilde{V}_{B_{2}^{h_{l}}}\right\|\right|}, \quad \forall i, k .
$$

Apply comparison principle 3.5 from subection 3.2, employing $U_{h_{l}}^{k}=W_{h_{l}}^{k}$, where $W_{h_{l}}^{k}$ is the grid function associated with the normalized contraction factor $\rho_{h_{l}}$ defined in (3.3) of subsection 3.4, and employing $\tilde{U}_{h_{l}}^{k}= \pm \tilde{W}_{h_{l}}^{k}$, where $\tilde{W}_{h_{l}}^{k}$ is defined above. The desired result follows immediately from the normalized contraction property.

Remark 7. Due to the stationarity of the discrete barrier function $w_{h_{l}}$, the upper bound for the contraction factor $\rho_{h_{l}}$ will be independent of $\tau_{l}$.

\section{MAXIMUM NORM STABILITY AND ACCURACY OF THE GLOBAL DISCRETIZATION}

In this section, we prove that the global discretization (2.4) is stable in the maximum norm and analyze its accuracy. We also show that the parallel Schwarz algorithm is geometrically convergent. The proofs are motivated by [32, 10] and employ Picard's contraction mapping theorem. In the first section, we describe the contraction mapping theorem and existence and uniqueness results for (2.4). We also discuss the geometric convergence of the parallel Schwarz iterates. In the second section we describe the stability of the global discretization. In the third section, we apply the stability theorem to estimate the accuracy of the global nonmatching grid discretization in terms of the local discretization and interpolation errors.

4.1. Contraction mapping theorem. The existence and uniqueness of solutions to the global discretized system (2.4) will be proved by applying Picard's contraction mapping theorem 3], which we summarize below for convenience. In addition, the geometric convergence of the parallel Schwarz iterates will follow from properties of the contraction mapping.

Theorem 4.1. Suppose the following hold.

1. Let $\mathcal{H}$ be a complete metric space with metric $d(.,$.$) .$

2. Let $\mathcal{T}: \mathcal{H} \rightarrow \mathcal{H}$ be a contractive mapping, i.e., for any $X, Y \in \mathcal{H}$ we have

$$
d(\mathcal{T} X, \mathcal{T} Y) \leq \delta d(X, Y),
$$

where $\delta<1$.

Then the following hold. 
1. There exists a unique fixed point $U \in \mathcal{H}$ of $\mathcal{T}$ satisfying

$$
U=\mathcal{T} U \text {. }
$$

2. Let $U^{(0)}$ be any element of $\mathcal{H}$. Then $U^{(n)} \equiv \mathcal{T}^{n} U^{(0)} \rightarrow U$ geometrically with

$$
\begin{aligned}
d\left(U^{(n+1)}, U\right) & \leq \delta d\left(U^{(n)}, U\right) \\
& \leq \delta^{n} d\left(U^{(0)}, U\right) .
\end{aligned}
$$

3. For any $U^{(0)} \in \mathcal{H}$ we have

$$
d\left(U^{(0)}, U\right) \leq \frac{1}{1-\delta} d\left(\mathcal{T} U^{(0)}, U^{(0)}\right) .
$$

Proof. See [3].

In our applications, we will choose the metric space $\mathcal{H}$ and mapping $\mathcal{T}$ so that global discretization (2.4) is a fixed point equation for $\mathcal{T}$. Furthermore, the parallel Schwarz iterates $\left\{U^{(n)}\right\}$, given a starting guess $U^{(0)}$, will correspond to $U^{(n+1)}=\mathcal{T} U^{(n)}$. For convenience, we will consider a system of equations more general than (2.4). Let $\tilde{f}_{h_{l}, I}^{k}$ and $\tilde{g}_{h_{l}, B_{2}}^{k}$ be given forcing terms for $k=0, \ldots, N_{l}$ and $l=1, \ldots, p$. Let $\tilde{u}_{h_{l}, I}^{0}$ be given initial data for $l=1, \ldots, p$. We consider the following general system of equations for unknowns $U_{h, \tau}=\left\{\left\{U_{h_{l}}^{k}\right\}_{k=0}^{N_{l}}\right\}_{l=1}^{p}$ :

$$
\begin{aligned}
\left(I+\tau_{l} \theta_{l} A_{I I}^{h_{l}}\right) U_{h_{l}, I}^{k+1}+\tau_{l} \theta_{l} A_{I B}^{h_{l}} U_{h_{l}, B}^{k+1}= & \left(I-\tau_{l} \tilde{\theta}_{l} A_{I I}^{h_{l}}\right) U_{h_{l}, I}^{k}-\tau_{l} \tilde{\theta}_{l} A_{I B}^{h_{l}} U_{h_{l}, B}^{k} \\
& +\tau_{l}\left(\theta_{l} \tilde{f}_{h_{l}, I}^{k+1}+\tilde{\theta}_{l} \tilde{f}_{h_{l}, I}^{k}\right), \\
U_{h_{l}, B_{1}}^{k+1}= & 0 \\
U_{h_{l}, B_{2}}^{k+1}= & \mathcal{I}_{h_{l}}^{k+1} U_{h, \tau}+\tilde{g}_{h_{l}, B_{2}}^{k+1}, \\
U_{h_{l}, I}^{0}= & \tilde{u}_{h_{l}, I}^{0},
\end{aligned}
$$

for $k=0, \ldots, N_{l}-1$ and $l=1, \ldots, p$.

Our choice for $\mathcal{H}$ will be based on the linear system (4.1). Given $\tilde{f}_{h_{l}, I}^{k}, \tilde{g}_{h_{l}, B_{2}}^{k}$ for $k=0, \ldots, N_{l}$ and $l=1, \ldots, p$ and $\tilde{u}_{h_{l}, I}^{0}$, we define

$$
\begin{aligned}
\mathcal{H} \equiv\{ & \left.X_{h, \tau}: X_{h_{l}}^{k} \text { satisfy following constraints }\right\} \\
\left(I+\tau_{l} \theta_{l} A_{I I}^{h_{l}}\right) X_{h_{l}, I}^{k+1}+\tau_{l} \theta_{l} A_{I B}^{h_{l}} X_{h_{l}, B}^{k+1}=\left(I-\tau_{l} \tilde{\theta}_{l} A_{I I}^{h_{l}}\right) X_{h_{l}, I}^{k}-\tau_{l} \tilde{\theta}_{l} A_{I B}^{h_{l}} X_{h_{l}, B}^{k} & +\tau_{l}\left(\theta_{l} \tilde{f}_{h_{l}, I}^{k+1}+\tilde{\theta}_{l} \tilde{f}_{h_{l}, I}^{k}\right), \\
X_{h_{l}, B_{1}}^{k+1}= & 0, \\
X_{h_{l}, I}^{0}= & \tilde{u}_{h_{l}, I}^{0},
\end{aligned}
$$

for $k=0, \ldots, N_{l}-1$ and $l=1, \ldots, p$. $\mathcal{H}$ is not a vector space due to the linear inhomogeneous constraints. However, $\mathcal{H}$ is closed in the vector space of all grid functions endowed with the maximum norm, and consequently, $\mathcal{H}$ will be a complete metric space if the metric $d(.,$.$) defined on \mathcal{H}$ is inherited from the standard maximum norm. Given $X_{h, \tau}, Y_{h, \tau} \in \mathcal{H}$, we define

$$
d\left(X_{h, \tau}, Y_{h, \tau}\right) \equiv\left|\left\|X_{h, \tau}-Y_{h, \tau}\right\|\right|,
$$


where $|\|\cdot\||$ denotes the maximum norm on the vector space of all space-time grid functions

$$
\left|\left\|X_{h, \tau}-Y_{h, \tau}\right\|\right| \equiv \max _{l=1, \ldots, p} \max _{k=0, \ldots, N_{l}} \max _{i \in \bar{\Omega}_{h_{l}}^{*}}\left|\left(X_{h_{l}}^{k}-Y_{h_{l}}^{k}\right)_{i}\right| .
$$

For the above $\mathcal{H}$, we define a mapping $\mathcal{T}: \mathcal{H} \rightarrow \mathcal{H}$ so that linear system (4.1) is a fixed point equation of $\mathcal{T}$ on $\mathcal{H}$. Given $X_{h, \tau} \in \mathcal{H}$ define $\tilde{X}_{h, \tau}=\mathcal{T} X_{h, \tau}$ as

$$
\begin{aligned}
\left(I+\tau_{l} \theta_{l} A_{I I}^{h_{l}}\right) \tilde{X}_{h_{l}, I}^{k+1}+\tau_{l} \theta_{l} A_{I B}^{h_{l}} \tilde{X}_{h_{l}, B}^{k+1}= & \left(I-\tau_{l} \tilde{\theta}_{l} A_{I I}^{h_{l}}\right) \tilde{X}_{h_{l}, I}^{k}-\tau_{l} \tilde{\theta}_{l} A_{I B}^{h_{l}} \tilde{X}_{h_{l}, B}^{k} \\
& +\tau_{l}\left(\theta_{l} \tilde{f}_{h_{l}, I}^{k+1}+\tilde{\theta}_{l} \tilde{f}_{h_{l}, I}^{k}\right), \\
\tilde{X}_{h_{l}, B_{1}}^{k+1}= & 0, \\
\tilde{X}_{h_{l}, B_{2}}^{k+1}= & \mathcal{I}_{h_{l}}^{k+1} X_{h, \tau}+\tilde{g}_{h_{l}, B_{2}}^{k+1}, \\
\tilde{X}_{h_{l}, I}^{0}= & \tilde{u}_{h_{l}, I}^{0},
\end{aligned}
$$

for $k=0, \ldots, N_{l}-1$ and $l=1, \ldots, p$. It immediately follows that system (4.1) is a fixed point equation of $\mathcal{T}$. Additionally, the parallel Schwarz iterates from Section 2 can be described in terms of the mapping $\mathcal{T}$ : Given a starting guess $U_{h, \tau}^{(0)}$ to (4.1), the subsequent parallel Schwarz iterates $\left\{U_{h, \tau}^{(n)}\right\}$ are

$$
U_{h, \tau}^{(n)} \equiv \mathcal{T} U_{h, \tau}^{(n-1)}, \quad n=1,2, \ldots
$$

The existence and uniqueness of solutions to system (4.1) and (2.4) will be guaranteed by Picard's contraction mapping theorem, provided $\mathcal{T}$ is a contraction mapping in the metric space $\mathcal{H}$. This contraction property of $\mathcal{T}$ is pivotal to the stability analysis in this paper and is proved next.

Theorem 4.2. Suppose the following hold.

1. Let matrix $A^{h_{l}}$ satisfy Assumptions $A 1$ and $A 5$ for $l=1, \ldots, p$.

2. Let $0<\tau_{l}$ satisfy the local stability criterion

$$
\tau_{l} \leq \min _{i \in I^{h_{l}}} \frac{1}{\tilde{\theta}_{l} A_{i i}^{h_{l}}}, \quad \text { if } \tilde{\theta}_{l} \neq 0,
$$

for $l=1, \ldots, p$.

3. Let the intergrid interpolation maps $\left\{\mathcal{I}_{h_{l}}^{k}\right\}_{k, l}$ satisfy Assumptions $A 2$ and A3.

4. Let the local overlap parameters $\beta_{l}$ be chosen large enough, and the local mesh size $h_{l} \leq h_{l}^{*}$ small enough so that the contraction factor $\rho_{h_{l}}$ satisfies

$$
\delta_{h} \equiv \sigma_{h, \tau} \max _{l} \rho_{h_{l}} \leq \delta<1,
$$

where $\sigma_{h, \tau}$ denotes the maximum norm of the map $\left\{\mathcal{I}_{h_{l}}^{k}\right\}_{k, l}$.

Then the following hold.

1. The mapping $\mathcal{T}$ will be a contraction mapping on $\mathcal{H}$ satisfying

$$
d\left(\mathcal{T} X_{h, \tau}, \mathcal{T} Y_{h, \tau}\right) \leq \delta d\left(X_{h, \tau}, Y_{h, \tau}\right), \quad \forall X_{h, \tau}, Y_{h, \tau} \in \mathcal{H} .
$$

2. System (4.1) will be uniquely solvable with a solution $U_{h, \tau}$. 
3. Given any starting guess $U_{h, \tau}^{(0)} \in \mathcal{H}$, the iterates $U_{h, \tau}^{(n)}=\mathcal{T}^{n} U_{h, \tau}^{(0)}$ (which correspond to the parallel Schwarz iterates) converge geometrically to the unique fixed point $U_{h, \tau}$ :

$$
\left|\left\|\mathcal{T}^{n} U_{h, \tau}^{(0)}-U_{h, \tau}\right\|\right| \leq \delta^{n}\left|\left\|U_{h, \tau}^{(0)}-U_{h, \tau}\right\|\right| .
$$

Proof. The proof is similar to [10] and relies on the maximum principle and the contraction property. Given $X_{h, \tau}, Y_{h, \tau} \in \mathcal{H}$, we need to estimate $d\left(\mathcal{T} X_{h, \tau}, \mathcal{T} Y_{h, \tau}\right)$ in terms of $d\left(X_{h, \tau}, Y_{h, \tau}\right)$. For convenience, denote $\tilde{X}_{h, \tau}=\mathcal{T} X_{h, \tau}$ and $\tilde{Y}_{h, \tau}=$ $\mathcal{T} Y_{h, \tau}$. By applying the definition of $\mathcal{T}$, we note that $\tilde{X}_{h, \tau}-\tilde{Y}_{h, \tau}$ will satisfy the homogeneous system

$$
\begin{aligned}
\left(I+\tau_{l} \theta_{l} A_{I I}^{h_{l}}\right)\left(\tilde{X}_{h_{l}, I}^{k+1}\right. & \left.-\tilde{Y}_{h_{l}, I}\right)+\tau_{l} \theta_{l} A_{I B}^{h_{l}}\left(\tilde{X}_{h_{l}, B}^{k+1}-\tilde{Y}_{h_{l}, B}^{k+1}\right) \\
& =\left(I-\tau_{l} \tilde{\theta}_{l} A_{I I}^{h_{l}}\right)\left(\tilde{X}_{h_{l}, I}^{k}-\tilde{Y}_{h_{l}, I}^{k}\right)+\tau_{l} \tilde{\theta}_{l} A_{I B}^{h_{l}}\left(\tilde{X}_{h_{l}, B}^{k}-\tilde{Y}_{h_{l}, B}^{k}\right), \\
\left(\tilde{X}_{h_{l}, B_{1}}^{k+1}-\tilde{Y}_{h_{l}, B_{1}}^{k+1}\right) & =0, \\
\left(\tilde{X}_{h_{l}, B_{2}}^{k+1}-\tilde{Y}_{h_{l}, B_{2}}^{k+1}\right) & =\mathcal{I}_{h_{l}}^{k+1}\left(X_{h, \tau}-Y_{h, \tau}\right), \\
\left(\tilde{X}_{h_{l}, I}^{0}-\tilde{Y}_{h_{l}, I}^{0}\right) & =0,
\end{aligned}
$$

for $k=0, \ldots, N_{l}-1$ and $l=1, \ldots, p$. By the maximum principle from Section 3 we obtain

$$
\begin{aligned}
\left|\left\|\tilde{X}_{h, \tau}-\tilde{Y}_{h, \tau}\right\|\right| & \leq \max _{k=0, \ldots, N_{l}} \max _{l=1, \ldots, p}\left|\mathcal{I}_{h_{l}}^{k}\left(X_{h, \tau}-Y_{h, \tau}\right)\right| \\
& \leq \sigma_{h, \tau} \max _{k=0, \ldots, N_{l}} \max _{l=1, \ldots, p} \max _{i \in \bar{\Omega}_{l}}\left|\left(X_{h_{l}}^{k}-Y_{h_{l}}^{k}\right)_{i}\right| .
\end{aligned}
$$

Here $\sigma_{h, \tau}$ is the maximum norm of the intergrid interpolation map $\mathcal{I}_{h_{l}}^{k}$.

Since $X_{h, \tau}, Y_{h, \tau} \in \mathcal{H}$, their difference $X_{h, \tau}-Y_{h, \tau}$ satisfies a discretized homogeneous parabolic equation, and by the contraction property, we obtain

$$
\max _{i \in \bar{\Omega}_{l}}\left|\left(X_{h_{l}}^{k}-Y_{h_{l}}^{k}\right)_{i}\right| \leq \rho_{h_{l}}\left|\left\|X_{h, \tau}-Y_{h, \tau}\right\|\right|,
$$

and consequently

$$
\max _{k=0, \ldots, N_{l}} \max _{l=1, \ldots, p} \max _{i \in \bar{\Omega}_{l}}\left|\left(X_{h_{l}}^{k}-Y_{h_{l}}^{k}\right)_{i}\right| \leq \max _{l=1, \ldots, p} \rho_{h_{l}}\left|\left\|X_{h, \tau}-Y_{h, \tau}\right\|\right| .
$$

Combining the two bounds, we obtain

$$
\begin{aligned}
\left|\left\|\tilde{X}_{h, \tau}-\tilde{Y}_{h, \tau}\right\|\right| & \leq \max _{k=0, \ldots, N_{l}} \max _{l=1, \ldots, p}\left|\mathcal{I}_{h_{l}}^{k}\left(X_{h, \tau}-Y_{h, \tau}\right)\right| \\
& \leq \sigma_{h, \tau} \max _{k=0, \ldots, N_{l} ; l=1, \ldots, p} \max _{i \in \bar{\Omega}_{l}}\left|\left(X_{h_{l}}^{k}-Y_{h_{l}}^{k}\right)_{i}\right| \\
& \leq \sigma_{h, \tau} \max _{l=1, \ldots, p} \rho_{h_{l}}\left|\left\|X_{h, \tau}-Y_{h, \tau}\right\|\right| \\
& =\delta \mid\left\|X_{h, \tau}-Y_{h, \tau}\right\| .
\end{aligned}
$$

Since $d\left(\tilde{X}_{h, \tau}, \tilde{Y}_{h, \tau}\right)=\left|\left\|\tilde{X}_{h, \tau}-\tilde{Y}_{h, \tau}\right\|\right|$ and $d\left(X_{h, \tau}, Y_{h, \tau}\right)=\left|\left\|X_{h, \tau}-Y_{h, \tau}|\||\right.\right.$, and since by assumption $\delta<1$, we obtain that $\mathcal{T}$ is a contraction

$$
d\left(\tilde{V}_{h, \tau}, \tilde{W}_{h, \tau}\right) \leq \delta d\left(V_{h, \tau}, W_{h, \tau}\right) .
$$


The unique solvability of the linear system follows from the uniqueness of the fixed point of a contraction mapping. The geometric convergence of the parallel Schwarz iterates follows by the geometric convergence of the Picard iterates.

We note that the preceding result does not directly provide an a priori bound for the maximum norm of the solution. This will be done in the next section.

4.2. Stability of the global discretization. In this section, we will derive an a priori bound for the maximum norm of the solution to 4.1.).

Theorem 4.3. Suppose the following hold.

1. Let matrices $A^{h_{l}}$ satisfy Assumptions $A 1$ and $A 5$ for $l=1, \ldots, p$.

2. Let $0<\tau_{l}$ satisfy the local stability constraint

$$
\tau_{l} \leq \min _{i \in I^{h_{l}}} \frac{1}{\tilde{\theta}_{l} A_{i i}^{h_{l}}}, \quad \text { if } \tilde{\theta}_{l} \neq 0
$$

for $l=1, \ldots, p$.

3. Let $\mathcal{I}_{h_{l}}^{k}$ satisfy Assumptions $A 2$ and A3.

4. Let the overlap parameters $\beta_{l}$ be chosen large enough, and the mesh sizes small enough with $h_{l} \leq h_{l}^{*}$ so that $\delta \equiv \sigma_{h, \tau} \max _{l} \rho_{h_{l}} \leq \delta<1$.

5. Let $U_{h, \tau}=\left\{U_{h_{l}}^{k}\right\}_{k, l}$ denote the unique solution of system (4.1).

Then, the maximum norm of the solution $U_{h, \tau}$ satisfies

$$
\begin{aligned}
\|\| U_{h, \tau} \| \mid \leq & \left(1+\frac{\sigma_{h, \tau}}{1-\delta}\right) \max _{l=1, \ldots, p}\left(\left\|\tilde{u}_{h_{l}, I}^{0}\right\|_{\infty, \Omega_{h_{l}}^{*}}+\tau_{l} \theta_{l}\left\|\tilde{f}_{h_{l}, I}^{N_{l}}\right\|_{\infty, \Omega_{h_{l}}^{*}}\right. \\
& \left.+\tau_{l} \sum_{k=1}^{N_{l}-1}\left\|\tilde{f}_{h_{l}, I}^{k}\right\|_{\infty, \Omega_{h_{l}}^{*}}+\tau_{l} \tilde{\theta}_{l}\left\|\tilde{f}_{h_{l}, I}^{0}\right\|_{\infty, \Omega_{h_{l}}^{*}}+\sum_{k=0}^{N_{l}}\left\|\tilde{g}_{h_{l}, B_{2}}^{k}\right\|_{\infty, B_{2}^{h_{l}}}\right) .
\end{aligned}
$$

Proof. Choose any suitable grid function $X_{h, \tau} \in \mathcal{H}$ and use it as an initial guess in the Picard fixed point iteration. By Theorem 4.1 (the contraction mapping)

$$
d\left(X_{h, \tau}, U_{h, \tau}\right) \leq \frac{1}{1-\delta} d\left(X_{h, \tau}, \mathcal{T} X_{h, \tau}\right)
$$

Using that the metric in $\mathcal{H}$ was inherited from the maximum norm, we obtain

$$
\begin{array}{rlrl}
\left|\left\|U_{h, \tau}\right\|\right| & \leq\left|\left\|X_{h, \tau}\right\|\right|+\mid\left\|U_{h, \tau}-X_{h, \tau}\right\|, & & \text { triangle inequality } \\
& =\left|\left\|X_{h, \tau}\right\|\right|+d\left(U_{h, \tau}, X_{h, \tau}\right) & \\
& =\left|\left\|X_{h, \tau}\right\|\right|+\frac{1}{1-\delta} d\left(X_{h, \tau}, \mathcal{T} X_{h, \tau}\right), & & \text { from above. }
\end{array}
$$

Therefore, to obtain a bound for $\left|\left\|U_{h, \tau}\right\|\right|$ we only need to choose $X_{h, \tau} \in \mathcal{H}$ and estimate $\left|\left\|X_{h, \tau}\right\|\right|$ and $d\left(X_{h, \tau}, \mathcal{T} X_{h, \tau}\right)$. 
Accordingly, choose $X_{h, \tau}=\left\{X_{h_{l}}^{k}\right\}_{k, l}$ as the solutions to the local discretized parabolic equations with trivial boundary conditions

$$
\begin{aligned}
\left(I+\tau_{l} \theta_{l} A_{I I}^{h_{l}}\right) X_{h_{l}, I}^{k+1}+\tau_{l} \theta_{l} A_{I B}^{h_{l}} X_{h_{l}, B}^{k+1}= & \left(I-\tau_{l} \tilde{\theta}_{l} A_{I I}^{h_{l}}\right) X_{h_{l}, I}^{k}-\tau_{l} \tilde{\theta}_{l} A_{I B}^{h_{l}} X_{h_{l}, B}^{k} \\
& +\tau_{l}\left(\theta_{l} \tilde{f}_{h_{l}, I}^{k+1}+\tilde{\theta}_{l} \tilde{f}_{h_{l}, I}^{k}\right), \\
X_{h_{l}, B_{1}}^{k+1}= & 0, \\
X_{h_{l}, B_{2}}^{k+1}= & 0, \\
X_{h_{l}, I}^{0}= & \tilde{u}_{h_{l}, I}^{0},
\end{aligned}
$$

for $k=0, \ldots, N_{l}-1$ and $l=1, \ldots, p$. Since the local problems for $\left\{X_{h_{l}}^{k}\right\}_{k}$ are decoupled, we can estimate the maximum norm of each local component $X_{h_{l}, \tau_{l}}$ independently by using the local a priori estimates from Section 3 . This yields

$$
\begin{aligned}
\left\|\left|X_{h, \tau} \|\right| \leq \max _{l=1, \ldots, p}\left(\left\|\tilde{u}_{h_{l}, I}^{0}\right\|_{\infty}+\tau_{l} \theta_{l}\left\|\tilde{f}_{h_{l}, I}^{N_{l}}\right\|_{\infty, \Omega_{h_{l}}^{*}}+\tau_{l} \sum_{k=1}^{N_{l}-1}\left\|\tilde{f}_{h_{l}, I}^{k}\right\|_{\infty, \Omega_{h_{l}}^{*}}\right.\right. \\
\left.+\tau_{l} \tilde{\theta}_{l}\left\|\tilde{f}_{h_{l}, I}^{0}\right\|_{\infty, \Omega_{h_{l}}^{*}}+\sum_{k=0}^{N_{l}}\left\|\tilde{g}_{h_{l}, B_{2}}^{k}\right\|_{\infty, B_{2}^{h_{l}}}\right) .
\end{aligned}
$$

We next estimate $\left|\left\|X_{h, \tau}-\mathcal{T} X_{h, \tau}\right\|\right|$. For convenience, let $\tilde{X}_{h, \tau}=\mathcal{T} X_{h, \tau}$. We note that $X_{h, \tau}-\tilde{X}_{h, \tau}$ will satisfy the discretized homogeneous parabolic equation

$$
\begin{aligned}
&\left(I+\tau_{l} \theta_{l} A_{I I}^{h_{l}}\right)\left(X_{h_{l}, I}^{k+1}-\tilde{X}_{h_{l}, I}^{k+1}\right)+\tau_{l} \theta_{l} A_{I B}^{h_{l}}\left(X_{h_{l}, B}^{k+1}-\tilde{X}_{h_{l}, B}^{k+1}\right) \\
&=\left(I-\tau_{l} \tilde{\theta}_{l} A_{I I}^{h_{l}}\right)\left(X_{h_{l}, I}^{k}-\tilde{X}_{h_{l}, I}^{k}\right)-\tau_{l} \tilde{\theta}_{l} A_{I B}^{h_{l}}\left(X_{h_{l}, B}^{k}-\tilde{X}_{h_{l}, B}^{k}\right), \\
&\left(X_{h_{l}, B_{1}}^{k+1}-\tilde{X}_{h_{l}, B_{1}}^{k+1}\right)=0 \\
&\left(X_{h_{l}, B_{2}}^{k+1}-\tilde{X}_{h_{l}, B_{2}}^{k+1}\right)=-\mathcal{I}_{h_{l}}^{k+1} X_{h, \tau}, \\
&\left(X_{h_{l}, I}^{0}-\tilde{X}_{h_{l}, I}^{0}\right)=0,
\end{aligned}
$$

for $k=0, \ldots, N_{l}-1$ and $l=1, \ldots, p$. By applying the discrete maximum principle and using $\sigma_{h, \tau}=\left|\left\|\mathcal{I}_{h_{l}}^{k}\right\|\right|$, we obtain

$$
\left|\left\|X_{h, \tau}-\tilde{X}_{h, \tau}\right\|\right| \leq \sigma_{h, \tau}\left|\left\|X_{h, \tau}\right\|\right| .
$$

Substituting these in our expression for $\left\||| U_{h, \tau}\right\| \mid$, we obtain

$$
\begin{aligned}
\left\|X_{h, \tau}\right\| \mid \leq & \left(1+\frac{\sigma_{h, \tau}}{1-\delta}\right) \max _{l=1, \ldots, p}\left(\left\|\tilde{u}_{h_{l}, I}^{0}\right\|_{\infty, \Omega_{h_{l}}^{*}}+\tau_{l} \theta_{l}\left\|\tilde{f}_{h_{l}, I}^{N_{l}}\right\|_{\infty, \Omega_{h_{l}}^{*}}\right. \\
& \left.+\tau_{l} \sum_{k=1}^{N_{l}-1}\left\|\tilde{f}_{h_{l}, I}^{k}\right\|_{\infty, \Omega_{h_{l}}^{*}}+\tau_{l} \tilde{\theta}_{l}\left\|\tilde{f}_{h_{l}, I}^{0}\right\|_{\infty, \Omega_{h_{l}}^{*}}+\sum_{k=0}^{N_{l}}\left\|\tilde{g}_{h_{l}, B_{2}}^{k}\right\|_{\infty, B_{2}}\right),
\end{aligned}
$$

which is the desired result. 
The above result depends critically on the contraction factor $\delta<1$. If the overlap $\beta_{l}$ of the local subregions are sufficiently large so that $\sigma_{h, \tau} \max _{l} \rho_{h_{l}} \leq \delta<1$ (uniformly in $h_{l}$ ) then the global discretization will be stable. In the next section, we apply the above stability result to estimate the accuracy of the nonmatching grid discretization (2.4).

4.3. Accuracy of the global discretization. From the general theory for discretization of linear evolution equations [30, we expect a stable and consistent scheme to be convergent. The same holds for the nonmatching overlapping grid discretization scheme (2.4) considered here. We will now consider the consistency of the global scheme (2.4), which is measured by the magnitude of the residual when $\pi_{h, \tau} u$ (i.e., the exact solution $u(x, t)$ restricted to the collection of space-time grids) is substituted into the scheme.

Definition. Given the restriction $u_{h, \tau}=\pi_{h, \tau} u$ of the exact solution $u(x, t)$ to the space-time grids, we define the grid function $\mathcal{E}_{h_{l}}^{k}(u)$ to represent the local discretization error on the grid $\Omega_{h_{l}}^{*}$ at time $k \tau_{l}$

$$
\begin{aligned}
\mathcal{E}_{h_{l}}^{k+1}(u) \equiv( & \left.+\tau_{l} \theta_{l} A_{I I}^{h_{l}}\right) u_{h_{l}, I}^{k+1}-\left(I-\tau_{l} \tilde{\theta} A_{I I}^{h_{l}}\right) u_{h_{l}, I}^{k} \\
& +\tau_{l}\left(\theta_{l} A_{I B}^{h_{l}} u_{h_{l}, B}^{k+1}+\tilde{\theta}_{l} A_{I B}^{h_{l}} u_{h_{l}, B}^{k}\right) \\
& -\tau_{l}\left(\theta_{l} f_{h_{l}, I}^{k+1}+\tilde{\theta}_{l} f_{h_{l}, I}^{k}\right) .
\end{aligned}
$$

We use $\mathcal{D}_{h_{l}}^{k}(u)$ to denote the boundary grid function representing the local intergrid interpolation error

$$
\mathcal{D}_{h_{l}}^{k}(u) \equiv u_{h_{l}, B_{2}}^{k+1}-\mathcal{I}_{h_{l}}^{k+1} u_{h, \tau}
$$

At each gridpoint $\left(x_{i}^{h_{l}}, k \tau_{l}\right)$, the local discretization error $\mathcal{E}_{h_{l}}^{k}(u)$ and the interpolation error $\mathcal{D}_{h_{l}}^{k}(u)$ can be estimated by expanding the stencils using Taylor series expansions centered at the gridpoint. The resulting estimate will involve the local mesh parameters $h_{l}$ and $\tau_{l}$, and higher order derivatives of $u(.,$.$) at one or more$ points in the convex hull of the gridpoints involved in that stencil. For convenience suppose that the discretization and interpolation errors satisfy

$$
\begin{aligned}
\left|\left(\mathcal{E}_{h_{l}}^{k}(u)\right)_{i}\right| & \leq C\|u\|_{q_{l ; 1}+2, q_{l ; 2}+1, \infty, \bar{\Omega}_{l}^{*} \times[0, T]}\left(h_{l}^{q_{l ; 1}}+\tau_{l}^{q_{l ; 2}}\right), \\
\left|\left(\mathcal{D}_{h_{l}}^{k}(u)\right)_{i}\right| & \leq C\|u\|_{r_{l ; 1}, r_{l ; 2}, \infty, B_{2}^{h_{l}, *} \times[0, T]}\left(h_{l}^{r_{l ; 1}}+\tau_{l}^{r_{l ; 2}}\right),
\end{aligned}
$$

where $B_{2}^{h_{l}, *}$ is a neighborhood of the boundary $B_{2}^{l}$ containing the union of all cells involved in the local intergrid interpolation.

We will now estimate the accuracy $\left|\left\|u_{h, \tau}-U_{h, \tau}\right\|\right|$ of the global discretization (2.4) in terms of the local discretization and interpolation errors, $\mathcal{E}_{h_{l}}^{k}(u)$ and $\mathcal{D}_{h_{l}}^{k}(u)$, respectively.

Theorem 4.4. Suppose the following hold.

1. Let matrices $A^{h_{l}}$ satisfy Assumptions $A 1$ and $A 5$ for $k=1, \ldots, p$. 
2. Let $0<\tau_{l}$ satisfy the following local stability constraint

$$
\tau_{l} \leq \min _{i \in I^{h_{l}}} \frac{1}{\tilde{\theta}_{l} A_{i i}^{h_{l}}}, \quad \text { if } \tilde{\theta}_{l} \neq 0,
$$

for $l=1, \ldots, p$.

3. Let $\mathcal{I}_{h_{l}}^{k}$ satisfy Assumptions $A 2$ and $A 3$.

4. Let the overlap parameter $\beta_{l}$ be chosen sufficiently large, and the mesh size sufficiently small with $h_{l} \leq h_{l}^{*}$ so that

$$
\delta \equiv \sigma_{h, \tau} \max _{l} \rho_{h_{l}}<1 .
$$

5. Let $U_{h, \tau}$ denote the solution to (2.4).

6. Let $u_{h, \tau}=\pi_{h, \tau} u$, the exact solution restricted to the grid, satisfy

$$
\begin{aligned}
\left(I+\tau_{l} \theta_{l} A_{I I}^{h_{l}}\right) u_{h_{l}, I}^{k+1}= & \left(I-\tau_{l} \tilde{\theta}_{l} A_{I I}^{h_{l}}\right) u_{h_{l}, I}^{k} \\
& +\tau_{l}\left(-\theta_{l} A_{I B}^{h_{l}} u_{h_{l}, I}^{k+1}+\tilde{\theta}_{l} A_{I B}^{h_{l}} u_{h_{l}, I}^{k}\right) \\
& +\tau_{l}\left(\theta_{l} f_{h_{l}, I}^{k+1}+\tilde{\theta}_{l} f_{h_{l}, I}^{k}\right) \\
& +\mathcal{E}_{h_{l}}^{k+1}(u) \\
u_{h_{l}, B_{1}}^{k+1}= & 0, \\
u_{h_{l}, B_{2}}^{k+1}= & \mathcal{I}_{h_{l}}^{k+1} u_{h, \tau}+\mathcal{D}_{h_{l}}^{k+1}(u), \\
u_{h_{l}, I}^{0}= & \pi_{h_{l}} u_{0},
\end{aligned}
$$

for $k=0, \ldots, N_{l}-1$ and $l=1, \ldots, p$, where $\mathcal{E}_{h_{l}}^{k}(u)$ and $\mathcal{D}_{h_{l}}^{k}(u)$ denote the local discretization and interpolation errors, respectively.

7. Let the local discretization and interpolation errors satisfy bounds (4.6).

Then $u_{h, \tau}-U_{h, \tau}$ satisfies the bounds

$$
\begin{aligned}
& \left|\left\|u_{h, \tau}-U_{h, \tau}\right\|\right| \leq\left(1+\frac{\sigma_{h, \tau}}{1-\delta}\right) \max _{l=1, \ldots, p}\left(\tau_{l} \theta_{l}\left\|\mathcal{E}_{h_{l}}^{N_{l}}(u)\right\|_{\infty, \bar{\Omega}_{h_{l}}^{*}}\right. \\
& +\tau_{l} \sum_{k=1}^{N_{l}-1}\left\|\mathcal{E}_{h_{l}}^{k}(u)\right\|_{\infty, \bar{\Omega}_{h_{l}}^{*}}+\tau_{l} \tilde{\theta}_{l}\left\|\mathcal{E}_{h_{l}}^{0}(u)\right\|_{\infty, \bar{\Omega}_{h_{l}}^{*}} \\
& \left.+\max _{k=0, \ldots, N_{l}}\left\|\mathcal{D}_{h_{l}}^{k}(u)\right\|_{\infty, B_{l, 2}^{*}}\right) \\
& \leq\left(1+\frac{\sigma_{h, \tau}}{1-\delta}\right) \max _{l=1, \ldots, p}\left(C\|u\|_{q_{1}+2, q_{2}+1, \infty, \bar{\Omega}_{l}^{*} \times[0, T]}\left(h_{l}^{q_{l ; 1}}+\tau_{l}^{q_{l ; 2}}\right)\right. \\
& \left.+C\|u\|_{r_{l ; 1}, r_{l ; 2}, \infty, B_{2}^{h_{l}, *} \times[0, T]}\left(h_{l}^{r_{l ; 1}}+\tau_{l}^{r_{l ; 2}}\right)\right) .
\end{aligned}
$$


Proof. Subtract equation (4.7) satisfied by $u_{h, \tau}$ from (2.4) satisfied by $U_{h, \tau}$. We obtain

$$
\begin{aligned}
\left(I+\tau_{l} \theta_{l} A_{I I}^{h_{l}}\right)\left(u_{h_{l}, I}^{k+1}-U_{h_{l}, I}^{k+1}\right)= & \left.\begin{array}{r}
I \\
-
\end{array} \tau_{l} \tilde{\theta}_{l} A_{I I}^{h_{l}}\right)\left(u_{h_{l}, I}^{k}-U_{h_{l}, I}^{k}\right) \\
& +\tau_{l}\left(-\theta_{l} A_{I B}^{h_{l}}\left(u_{h_{l}, I}^{k+1}-U_{h_{l}, I}^{k+1}\right)\right. \\
& \left.+\tilde{\theta}_{l} A_{I B}^{h_{l}}\left(u_{h_{l}, I}^{k}-U_{h_{l}, I}^{k}\right)\right) \\
& +\mathcal{E}_{h_{l}}^{k+1}(u) \\
\left(u_{h_{l}, B_{1}}^{k+1}-U_{h_{l}, B_{1}}^{k+1}\right)= & 0, \\
\left(u_{h_{l}, B_{2}}^{k+1}-U_{h_{l}, B_{2}}^{k+1}\right)= & \mathcal{I}_{h_{l}}^{k+1}\left(u_{h, \tau}-U_{h, \tau}\right)+\mathcal{D}_{h_{l}}^{k+1}(u), \\
\left(u_{h_{l}, I}^{0}-U_{h_{l}, I}^{0}\right)= & 0,
\end{aligned}
$$

for $k=0, \ldots, N_{l}-1$ and $l=1, \ldots, p$.

Since the hypothesis of the stability theorem from the preceding section is satisfied, we may apply it with $\tilde{f}_{h_{l}, I}^{k}=\mathcal{E}_{h_{l}}^{k}(u)$ and $\tilde{g}_{h_{l}}^{k}=\mathcal{D}_{h_{l}}^{k}(u)$ to obtain

$$
\begin{aligned}
&\left\|u_{h, \tau}-U_{h, \tau}\right\| \mid \leq\left(1+\frac{\sigma_{h, \tau}}{1-\delta}\right) \max _{l=1, \ldots, p}\left(\tau_{l} \theta_{l}\left\|\mathcal{E}_{h_{l}}^{N_{l}}(u)\right\|_{\infty, \bar{\Omega}_{h_{l}}^{*}}\right. \\
&+\tau_{l} \sum_{k=1}^{N_{l}-1}\left\|\mathcal{E}_{h_{l}}^{k}(u)\right\|_{\infty, \bar{\Omega}_{h_{l}}^{*}}+\tau_{l} \tilde{\theta}_{l}\left\|\mathcal{E}_{h_{l}}^{0}(u)\right\|_{\infty, \bar{\Omega}_{h_{l}}^{*}} \\
&\left.+\max _{k=0, \ldots, N_{l}}\left\|\mathcal{D}_{h_{l}}^{k}(u)\right\|_{\infty, B_{l, 2}^{*}}\right) .
\end{aligned}
$$

Substituting the bounds (4.6) for the local discretization and interpolation errors, we obtain

$$
\begin{aligned}
& \|\| u_{h, \tau}-U_{h, \tau} \| \mid \\
& \leq\left(1+\frac{\sigma_{h, \tau}}{1-\delta}\right) \max _{l=1, \ldots, p}\left(C\|u\|_{q_{l ; 1}+2, q_{l ; 2}+1, \infty, \bar{\Omega}_{l}^{*} \times[0, T]}\left(h_{l}^{q_{l ; 1}}+\tau_{l}^{q_{l ; 2}}\right)\right. \\
& \left.\quad+C\|u\|_{r_{l ; 1}, r_{l ; 2}, \infty, B_{2}^{h_{l}, *} \times[0, T]}\left(h_{l}^{r_{l ; 1}}+\tau_{l}^{r_{l ; 2}}\right)\right),
\end{aligned}
$$

which is the desired result.

Remark 8. The above global error bound provides some guidance on the selection of the local mesh sizes. If a global error of $O(\epsilon)$ is desired, then $h_{l}, \tau_{l}$ and $\mathcal{I}_{h_{l}}^{k}$ should be chosen so that

$$
\begin{aligned}
\epsilon & \approx\|u\|_{r_{1}, r_{2}, \infty, B_{2}^{h_{l}, *} \times[0, T]}\left(h_{l}^{r_{l ; 1}}+\tau_{l}^{r_{l ; 2}}\right) \\
& \approx\|u\|_{q_{l ; 1}+2, q_{l ; 2}+1, \infty, \bar{\Omega}_{l}^{*} \times[0, T]}\left(h_{l}^{q_{l ; 1}}+\tau_{l}^{q_{l ; 2}}\right),
\end{aligned}
$$

for $l=1, \ldots, p$. Thus, the mesh sizes should be smaller (or the interpolation stencil, of higher order) in regions where the solution is less smooth.

Remark 9. We assumed throughout this paper that $c(x) \geq c_{0}>0$, in order to guarantee the stability of the global discretization (2.4). In practice, however, the discretization considered should be stable and convergent even if this condition is 
violated, though the authors do not have rigorous results on this. We illustrate this by a simple example. Consider the parabolic equation

$$
\left\{\begin{aligned}
u_{t}-u_{x x} & =f(x, t), & & \text { in }(0,1) \times[0, T], \\
u(x, t) & =0, & & \text { on }\{0\} \times[0, T], \\
u(x, t) & =0, & & \text { on }\{1\} \times[0, T], \\
u(x, 0) & =u_{0}(x), & & \text { on }(0,1) .
\end{aligned}\right.
$$

Choose the following subdomains $\Omega_{1}=(0,1 / 3), \Omega_{2}=(1 / 3,2 / 3), \Omega_{3}=(2 / 3,1)$ and the following extended subdomains $\Omega_{1}^{*}=(0,1 / 2), \Omega_{2}^{*}=(1 / 4,3 / 4)$ and $\Omega_{3}^{*}=$ $(1 / 2,1)$. Since the associated elliptic operator is $L u=-u_{x x}$, we can solve local homogeneous problems explicitly to obtain the estimates for the local contraction factors

$$
\rho_{1}=\frac{1}{6}, \quad \rho_{2}=1, \quad \rho_{3}=\frac{1}{6} .
$$

We note that $\rho_{2}=1$ since on subdomain $\Omega_{2}^{*}, u(x) \equiv 1$ is the solution to the homogeneous elliptic equation $-u_{x x}=0$ with boundary conditions $u(1 / 4)=1$ and $u(3 / 4)=1$. Thus, the condition $\delta=\sigma_{h, \tau} \max _{l} \rho_{l}<1$ will be violated, and the mapping $\mathcal{T}$ will not be a contraction. However, it can easily be verified that the mapping $\mathcal{T}^{2}$ is a contraction with contraction factor $1 / 6$, and the global scheme should be stable. More generally, when $c(x)=0$, we expect the subdomains adjacent to the boundary $\partial \Omega$ to have contraction factors less than 1 and interior (floating) subdomains to have contraction factors of 1 . Repeated applications of $\mathcal{T}$ should however "propagate" the contraction property to the interior subdomains, and $\mathcal{T}^{k_{0}}$ may be contractive for some $k_{0}>1$. Unfortunately, rigorous results along these lines are not known to the authors.

Remark 10. We indicate briefly how the results of this paper can be extended to certain semilinear reaction diffusion equations. Consider the equation

$$
\left\{\begin{aligned}
u_{t}-\Delta u+\vec{b}(x) \cdot \nabla u+c(x, u) & =f(x, t), & & \text { in } \Omega \times[0, T], \\
u(x, t) & =0, & & \text { on } \partial \Omega \times[0, T], \\
u(x, 0) & =u_{0}(x), & & \text { on }(0,1),
\end{aligned}\right.
$$

where $c(x, u)$ is a smooth function and satisfies

$$
\frac{\partial c}{\partial u}(x, u) \geq c_{0}>0, \quad \forall x, u .
$$

On each local grid, we will discretize the semilinear elliptic operator

$$
L(u) \equiv-\Delta u+\vec{b}(x) \cdot \nabla u+c(x, u)
$$

using finite difference schemes, and discretize the parabolic equation in time using a $\theta$-scheme. Due to the nonlinearity $c(x, u)$, the following approximation will be used to avoid solving a nonlinear equation at each time step

$$
c\left(x, U_{h_{l}}^{k+1}\right) \approx c\left(x, U_{h_{l}}^{k}\right)+c_{u}\left(x, U_{h_{l}}^{k}\right)\left(U_{h_{l}}^{k+1}-U_{h_{l}}^{k}\right) .
$$


This will correspond to applying one-step of a Newton approximation and the resulting $\theta$-scheme approximation of the semilinear terms on $\left[k \tau_{l},(k+1) \tau_{l}\right]$ will be:

$$
\begin{aligned}
\theta_{l} c\left(x_{i}^{h_{l}},\left(U_{h_{l}}^{k+1}\right)_{i}\right)= & \\
+\tilde{\theta}_{l} c\left(x_{i}^{h_{l}},\left(U_{h_{l}}^{k}\right)_{i}\right) \approx & \theta_{l}\left\{c\left(x_{i}^{h_{l}},\left(U_{h_{l}}^{k}\right)_{i}\right)+c_{u}\left(x_{i}^{h_{l}},\left(U_{h_{l}}^{k}\right)_{i}\right)\left(\left(U_{h_{l}}^{k+1}-U_{h_{l}}^{k}\right)_{i}\right)\right\} \\
& +\tilde{\theta}_{l} c\left(x_{i}^{h_{l}},\left(U_{h_{l}}^{k}\right)_{i}\right) \\
= & c\left(x_{i}^{h_{l}},\left(U_{h_{l}}^{k}\right)_{i}\right) \\
& +\tilde{\theta}_{l} c_{u}\left(x_{i}^{h_{l}},\left(U_{h_{l}}^{k}\right)_{i}\right)\left(\left(U_{h_{l}}^{k+1}-U_{h_{l}}^{k}\right)_{i}\right) .
\end{aligned}
$$

Due to the above linearization and the sign properties of $c_{u}(.,$.$) , the existence of$ solutions of the local $\theta$-discretizations are guaranteed. Provided the local time steps $\tau_{l}$ are small enough to satisfy the local stability conditions (which would depend on the $c_{u}(.,$.$) terms), the theory developed in this paper can be extended to analyze$ the accuracy of the global discretization of the semilinear parabolic equation. The local truncation error terms will be different from the linear case and the local interpolation error will be identical to the linear case. We omit the details.

Remark 11. To simplify our discussions, we had assumed throughout the paper that the diffusion term was $a \Delta u$. More generally, the results of this paper are valid even if the diffusion term is of the form $\nabla \cdot(a(x) \nabla u)$, where $a(x)$ is a diagonal matrix with positive diagonal entries, provided the spatial discretization yields an $M$-matrix. In particular, if $a(x)$ is zero on a subregion (or on all of $\Omega$ ) the results would still hold (provided the discretization yields an $M$-matrix, which, due to the hyperbolic nature of the equation, would necessitate that a first order upwind discretization be used to discretize $\vec{b}(x) \cdot \nabla u)$. Consider now the original parabolic equation with $a \ll 1$ (i.e., a singularly perturbed parabolic equation). Suppose $\Omega_{1}^{*}$ and $\Omega_{2}^{*}$ are two overlapping subregions covering $\Omega$, such that

$$
|a \Delta u| \leq \eta, \quad \text { on } \Omega_{1}^{*} \times[0, T], \quad \text { where } \eta \ll 1 .
$$

If $a \Delta u$ is dropped on $\Omega_{1}^{*} \times[0, T]$, the resulting hyperbolic equation may be discretized by an explicit scheme (with suitable restriction on the time step) without requiring the solution of a linear system. A coupled hyperbolic-parabolic problem may be constructed to approximate the original parabolic equation (see for instance [29, 11]), as indicated next. Let $B_{1}^{i}=\partial \Omega_{i}^{*} \cap \partial \Omega$ and $B_{2}^{i}=\partial \Omega_{i}^{*} \cap \Omega$ for $i=1,2$, and let $B_{j, i n}^{1}$ denote the inflow segment of $B_{j}^{1}$. Additionally, let $v$ and $w$ denote the approximations of $u$ on $\Omega_{1}^{*} \times[0, T]$ and $\Omega_{2}^{*} \times[0, T]$, respectively. A coupled hyperbolic-parabolic system for $v$ and $w$ can be posed as follows:

$$
\left\{\begin{aligned}
v_{t}+\vec{b}(x) \cdot \nabla v+c(x) v & =f(x), & & \text { in } \Omega_{1}^{*} \times[0, T], \\
v & =0, & & \text { on } B_{1, i n}^{1} \times[0, T], \\
v & =w, & & \text { on } B_{2, i n}^{1} \times[0, T], \\
v & =u_{0}(x), & & \text { or } t=0
\end{aligned}\right.
$$

and

$$
\left\{\begin{aligned}
w_{t}-a \Delta w+\vec{b}(x) \cdot \nabla w+c(x) w & =f(x), & & \text { in } \Omega_{2}^{*} \times[0, T], \\
w & =0, & & \text { on } B_{1}^{2} \times[0, T], \\
w & =v, & & \text { on } B_{2} \times[0, T], \\
w & =u_{0}(x), & & \text { for } t=0 .
\end{aligned}\right.
$$


Provided the $M$-matrix assumption holds for the local discretizations, Theorem 4.4 would guarantee the maximum norm stability of the global nonmatching overset grid discretization of the above system. If $u$ denotes the solution of the original parabolic equation (with $u_{h, \tau}$ denoting its restriction to the space-time grids) and if $\left(v_{h_{1}, \tau_{1}}, w_{h_{2}, \tau_{2}}\right)$ denotes the discrete hyperbolic-parabolic solution, then the error $u_{h, \tau}-\left(v_{h_{1}, \tau_{1}}, w_{h_{2}, \tau_{2}}\right)$ will satisfy (4.8) with an additional term of magnitude $\eta$ on $\Omega_{1}^{*} \times[0, T]:$

$$
\begin{aligned}
& \left|\left\|u_{h, \tau}-\left(v_{h_{1}, \tau_{1}}, w_{h_{2}, \tau_{2}}\right)\right\|\right| \\
& \leq\left(1+\frac{\sigma_{h, \tau}}{1-\delta}\right) \max _{l=1, \ldots, p}\left(C\|u\|_{q_{l ; 1}+2, q_{l ; 2}+1, \infty, \bar{\Omega}_{l}^{*} \times[0, T]}\left(h_{l}^{q_{l ; 1}}+\tau_{l}^{q_{l ; 2}}\right)\right. \\
& \left.\quad+C\|u\|_{r_{l ; 1}, r_{l ; 2}, \infty, B_{2}^{h_{l}, *} \times[0, T]}\left(h_{l}^{r_{l ; 1}}+\tau_{l}^{r_{l ; 2}}\right)+\eta\right) .
\end{aligned}
$$

Thus, if $\eta$ is of the same magnitude as the local truncation and interpolation errors, then

$$
||\left|u_{h, \tau}-\left(v_{h_{1}, \tau_{1}}, w_{h_{2}, \tau_{2}}\right)\left\||\approx||| u_{h, \tau}-\left(U_{h_{1}, \tau_{1}}, U_{h_{2}, \tau_{2}}\right)\right\|\right|
$$

where $\left(U_{h_{1}, \tau_{1}}, U_{h_{2}, \tau_{2}}\right)$ is the nonmatching overset grid solution of the full parabolic equation. The domain $\Omega_{1}^{*}$ may be adaptively determined as in the $\chi$-formulation (see [8]). We omit further details.

\section{ACKNOWLEDGMENTS}

The authors wish to thank the Consiglio Nazionale delle Recerche of Italy for support during the visit of the first author to the Università dell'Aquila.

\section{REFERENCES}

1. G. Abdoulaev, Y. Achdou, J. Hontand, Y. Kuznetsov, O. Pironneau, and C. Prud'homme, Nonmatching grids for fluids, The tenth international conference on domain decomposition methods for partial differential equations (Providence, R.I) (J. Mandel, C. Farhat, and X.-C. Cai, eds.), AMS, 1998. MR 99i:76103

2. Y. Achdou, Y. Maday, and O. Widlund, Iterative substructuring preconditioners for mortar element methods in two dimensions, Tech. report, TR-735, Department of Computer Science, Courant Institute of Mathematical Sciences, 1997; SIAM J. Numer. Anal. 36 (1999), 551-580. MR 99m:65233

3. V. I. Arnold, Ordinary differential equations, Springer-Verlag, New York, 1992. MR 93b:34001

4. F. Ben Belgacem, The mortar finite element method with Lagrange multipliers, Numer. Math. 84 (1999), 173-197.

5. C. Bernardi, Y. Maday, and A. Patera, A new nonconforming approach to domain decomposition: The mortar element method, College de France Seminar (H. Brezis and J. L. Lions, eds.), Pitman, 1990. MR 95a:65201

6. C. Börgers and C. S. Peskin, A Lagrangian fractional step method for the incompressible Navier-Stokes equations on a periodic domain, J. Comp. Phys. 70 (1987), no. 2, 397-438. MR 89c:76007

7. J. H. Bramble, R. E. Ewing, J.E. Pasciak, and A. H. Schatz, A preconditioning technique for the efficient solution of problems with local grid refinement, Comput. Meth. Appl. Mech. Engrg. 67 (1988), 149-159.

8. F. Brezzi, C. Canuto, and A. Russo, A self-adaptive formulation for the Euler/Navier-Stokes coupling, Comp. Meth. in Appl. Mech. Engrg. 73 (1989), 317-330. MR 90h:76075

9. X. C. Cai, M. Dryja, and M. Sarkis, Overlapping nonmatching grids mortar element methods for elliptic problems I: Error analysis, SIAM J. Numer. Anal. (2000), 36 (1999), 581-606. MR 2000a: 65142 
10. X. C. Cai, T. P. Mathew, and M. Sarkis, Maximum norm analysis of overlapping nonmatching grid discretizations of elliptic equations, SIAM J. Numer. Anal. 37 (2000), no. 5, 1709-1728. MR 2001c:65130

11. C. Canuto and A. Russo, Self-adaptive coupling of mathematical models and/or numerical methods, Contemporary Mathematics 157 (1994), 35-44. MR 94m:35085]

12. T. F. Chan and T. P. Mathew, Domain decomposition algorithms, Acta Numerica (1994), 61-143. MR 95f:65214

13. G. Chesshire and W. D. Henshaw, Composite overlapping meshes for the solution of partial differential equations, J. Comp. Phys. 90 (1990), 1-64.

14. P. G. Ciarlet, Discrete maximum principle for finite-difference operators, Aequationes Math. 4 (1970), 338-352. MR 45:1404

15. M. Dryja and O. B. Widlund, An additive variant of the Schwarz alternating method for the case of many subregions, Tech. Report 339, also Ultracomputer Note 131, Department of Computer Science, Courant Institute, 1987.

16. - Domain decomposition algorithms with small overlap, SIAM J. Sci. Comp. 15 (1994), no. 3, 604-620. MR 95d:65102

17. R. E. Ewing, Domain decomposition techniques for efficient adaptive local grid refinement, Domain Decomposition Methods (Philadelphia, PA) (Tony Chan, Roland Glowinski, Jacques Périaux, and Olof Widlund, eds.), SIAM, 1989. MR 90c:65141

18. R. E. Ewing, R. D. Lazarov, and P. S. Vassilevski, Local refinement techniques for elliptic problems on cell-centered grids, Tech. report, University of Wyoming, 1988.

19. C. Farhat, J. Mandel, and F. X. Roux, Optimal convergence properties of the FETI domain decomposition method, Comp. Meth. Appl. Mech. Engrg. 115 (1994), 365-385. MR 95d:65091

20. P. J. J. Ferket and A. A. Reusken, A finite difference discretization method for elliptic problems on composite grids, Computing 56 (1996), 343-369. MR 97c:65174

21. M. Garbey, Y. Kuznetsov, and Y. Vassilevski, Parallel Schwarz methods for advectiondiffusion equations, SIAM J. Sci. Comp. 22-3, (2000), 891-916.

22. E. Giladi and H. B. Keller, Space-time domain decomposition for parabolic problems, Proceedings of IMACS conference (J. Wang, M. Allen, B. Chen, and T. Mathew, eds.), 1997, Also appeared as technical report from Center for Research on Parallel Computation, CRPCTR97701.

23. W. D. Henshaw, Automatic grid generation, Acta Numerica (1996), 121-148. CMP 98:14

24. C. Johnson, Numerical solutions of partial differential equations by the finite element method, Cambridge University Press, Cambridge, 1987. MR 89b:65003a and MR 89b:65003b

25. A. Karafiat, Discrete maximum principle in parabolic boundary value problems, Ann. Polon. Math. 53 (1991), 253-265. MR 92d:35052

26. P. L. Lions, On the Schwarz alternating method. I., First International Symposium on Domain Decomposition Methods for Partial Differential Equations (Philadelphia, PA) (Roland Glowinski, Gene H. Golub, Gérard A. Meurant, and Jacques Périaux, eds.), SIAM, 1988. MR 90a:65248

27. - On the Schwarz alternating method. II., Domain Decomposition Methods (Philadelphia, PA) (Tony Chan, Roland Glowinski, Jacques Périaux, and Olof Widlund, eds.), SIAM, 1989. MR 90e:65140

28. T. P. Mathew, Uniform convergence of the Schwarz alternating method for solving singularly perturbed advection diffusion equations, SIAM J. Numer. Anal. 35 (1998), no. 4, 1663-1683. MR 99f:65156

29. A. Quarteroni and A. Valli, Theory and applications of Steklov-Poincaré operators for boundary-value problems: the heterogeneous operator case, Proceedings of 4th International Conference on Domain Decomposition Methods, Moscow (Philadelphia) (Tony Chan, Roland Glowinski, Jacques Périaux, and Olof Widlund, eds.), SIAM, 1990. MR 92g:65132

30. R. D. Richtmyer and K. W. Morton, Difference methods for initial-value problems, Wiley Interscience, New York, 1967. MR 36:3515

31. Y. Saad, Iterative methods for sparse linear systems, PWS Publishing Company, Boston, MA, U.S.A, 1996

32. G. Starius, Composite mesh difference methods for elliptic boundary value problems, Numer. Math. 28 (1977), 243-258. MR 57:1923

33. J. Steger and J. Benek, On the use of composite grid schemes in computational aerodynamics, Comp. Meth. Appl. Mech. Engrg. 64 (1987), 301-320. MR 88i:65146 
34. R. S. Varga, Matrix iterative analysis, Prentice-Hall, 1962. MR 28:1725

35. J. Xu, Iterative methods by space decomposition and subspace correction, SIAM Review 34 (1992), 581-613. MR 93k:65029

115 Seal Rock Drive, San Francisco, California 94121

E-mail address: tmathew@mindspring.com

Dipartimento di Matematica ed Informatica, Università di Catania, Viale Andrea Doria 6, 95125 Catania, Italy

E-mail address: russo@dmi.unict.it 